\title{
Best Practices of Blood Cultures in Low- and Middle-Income Countries
}

\author{
Sien Ombelet ${ }^{1,2 *}$, Barbara Barbé ${ }^{1}$, Dissou Affolabi ${ }^{3}$, Jean-Baptiste Ronat ${ }^{4}$, \\ Palpouguini Lompo ${ }^{5}$, Octavie Lunguya ${ }^{6,7}$, Jan Jacobs ${ }^{1,2}$ and Liselotte Hardy ${ }^{1}$ \\ ${ }^{1}$ Department of Clinical Sciences, Institute of Tropical Medicine, Antwerp, Belgium, ${ }^{2}$ Department of Microbiology and \\ Immunology, KULeuven, Leuven, Belgium, ${ }^{3}$ Centre National Hospitalier Universitaire-Hubert Koutoucou Maga, Cotonou, \\ Benin, ${ }^{4}$ Médecins Sans Frontières, Operational Center Paris, Paris, France, ${ }^{5}$ Clinical Research Unit of Nanoro, Institut de \\ Recherche en Science de la Santé, Nanoro, Burkina Faso, ${ }^{6}$ National Institute for Biomedical Research, Kinshasa, Democratic \\ Republic of the Congo, ${ }^{7}$ Department of Medical Biology, Cliniques Universitaires, Université de Kinshasa, Kinshasa, \\ Democratic Republic of the Congo
}

OPEN ACCESS

Edited by:

Zisis Kozlakidis, International Agency for Research on

Cancer (IARC), France

Reviewed by:

Denise Myriam Dekker,

Bernhard-Nocht-Institut Für

Tropenmedizin (BMITM), Germany

Elodie Caboux

International Agency for Research on

Cancer (IARC), France

*Correspondence:

Sien Ombelet

sombelet@itg.be

Specialty section:

This article was submitted to Infectious Diseases - Surveillance,

Prevention and Treatment,

a section of the journal

Frontiers in Medicine

Received: 08 February 2019

Accepted: 29 May 2019

Published: 18 June 2019

Citation:

Ombelet $S$, Barbé B, Affolabi $D$, Ronat J-B, Lompo P, Lunguya $O$, Jacobs J and Hardy L (2019) Best Practices of Blood Cultures in Lowand Middle-Income Countries.

Front. Med. 6:131.

doi: 10.3389/fmed.2019.00131
Bloodstream infections (BSI) have a substantial impact on morbidity and mortality worldwide. Despite scarcity of data from many low- and middle-income countries (LMICS), there is increasing awareness of the importance of BSI in these countries. For example, it is estimated that the global mortality of non-typhoidal Salmonella bloodstream infection in children under 5 already exceeds that of malaria. Reliable and accurate diagnosis of these infections is therefore of utmost importance. Blood cultures are the reference method for diagnosis of BSI. LMICs face many challenges when implementing blood cultures, due to financial, logistical, and infrastructure-related constraints. This review aims to provide an overview of the state-of-the-art of sampling and processing of blood cultures, with emphasis on its use in LMICs. Laboratory processing of blood cultures is relatively straightforward and can be done without the need for expensive and complicated equipment. Automates for incubation and growth monitoring have become the standard in high-income countries (HICs), but they are still too expensive and not sufficiently robust for imminent implementation in most LMICs. Therefore, this review focuses on "manual" methods of blood culture, not involving automated equipment. In manual blood cultures, a bottle consisting of a broth medium supporting bacterial growth is incubated in a normal incubator and inspected daily for signs of growth. The collection of blood for blood culture is a crucial step in the process, as the sensitivity of blood cultures depends on the volume sampled; furthermore, contamination of the blood culture (accidental inoculation of environmental and skin bacteria) can be avoided by appropriate antisepsis. In this review, we give recommendations regarding appropriate blood culture sampling and processing in LMICs. We present feasible methods to detect and speed up growth and discuss some challenges in implementing blood cultures in LMICs, such as the biosafety aspects, supply chain and waste management.

Keywords: clinical bacteriology, blood culture, low-resource settings (LRS), laboratory medicine practices, bacteremia diagnosis 


\section{INTRODUCTION}

\section{Scope of This Review}

This review provides an overview of current best practices in sampling and processing blood cultures in low- and middleincome countries (LMICs). LMICs are defined depending on gross national income per capita by the World Bank ${ }^{1}$. LMICs face many challenges when implementing laboratory medicine, related to lack of financial and human resources and infrastructure (1). Since most studies on blood cultures have been performed in high-income countries (HICs), many recommendations from these studies cannot be easily adopted in LMICs. This review will therefore focus on blood culture methods and techniques appropriate for settings with limited resources. Studies conducted in LMICs will be mentioned explicitly when available.

Furthermore, attention will be given to caveats and obstacles of implementing blood cultures in LMICs. The details of implementing such a blood culture system in microbial surveillance and techniques used for identification and antibiotic susceptibility testing will not be discussed. In addition, advice on implementation of quality management for clinical bacteriology in LMICs has already been described elsewhere (2).

\section{Blood Culture Definitions and Work-Flow}

In normal conditions, blood is sterile. Severe localized or systemic infections can cause micro-organisms to enter the bloodstream through the lymphatic system. This presence of bacteria in the bloodstream is called "bacteremia." Most of the time, these bacteria are cleared quickly by the immune system. In the case of overwhelming infections or intravascular focus of infection, the immune system may be unable to clear the bacteria from the blood, resulting in a bloodstream infection (BSI) (3). The micro-organisms responsible for this infection can be identified by blood culture. A blood culture consists of a blood sample from a patient, suspected to have a BSI, which is inoculated into a specialized blood culture bottle containing a broth (i.e., liquid) medium that supports optimal growth of bacteria. The concentration of bacteria in the blood of patients with BSI is very low (4), therefore direct culture on an agar plate cannot detect the presence of bacteria in the patient's blood. Once the blood is inoculated into the blood culture bottle, further amplification of the bacteria can take place, ultimately leading to visible bacterial growth. When growth of bacteria is detected in the blood culture bottle, a Gram stain of the blood-broth mixture is done to confirm presence of micro-organisms and distinguish between Gram-positive, Gram-negative bacteria and yeasts (Table 1). This and other microscopic information, such as shape and configuration of the bacteria, can orient the clinician to the identification of the bacteria causing the BSI and hence to the most appropriate antibiotic treatment. A subculture of the blood-broth mixture on an agar plate is then done to obtain colonies of the pathogen, on which further identification and antibiotic susceptibility testing can be performed. See Figure 1

\footnotetext{
${ }^{1}$ https://blogs.worldbank.org/opendata/new-country-classifications-incomelevel-2018-2019
}

for a visualization of the blood culture workflow and Table 2 for definitions regarding blood cultures.

\section{Indications for Blood Culture}

Blood cultures must be obtained whenever there is a clinical suspicion of BSI. However, it is currently not clear which clinical signs are good predictors for BSI. Many predictive models to optimize the yield of blood cultures have been proposed, but so far only two of these have been shown to reliably distinguish between high $(>30 \%)$ and low $(<3 \%)$ risk of BSI, according to a 2015 systematic review (8). Of these, one model is a computerized system using a causal probabilistic network with input of many different variables (9), compromising its possible use in LMICs, and the other has been validated only for patients with community-acquired pneumonia (10). Moreover, none of these validated models are used in routine clinical practice, possibly because they are too complicated to calculate at the bedside and because of their reliance on laboratory data that are not available at the time of blood sampling (8).

Other well-studied clinical predictive models are the SIRS criteria (systemic inflammatory response syndrome) and the Shapiro criteria $(11,12)$ (see Figure 2). Both these models have demonstrated high sensitivity but low specificity (14-16) and rely on laboratory parameters that are not readily available in most LMICs. Their usefulness in daily practice is therefore rather limited.

In 2016, the Third International Consensus Definitions Task force described an easy clinical score to identify patients at risk of sepsis, the quick Sequential Organ Failure Assessment (qSOFA) score (13) (see Figure 2). Sepsis is defined as life-threatening organ dysfunction caused by a dysregulated host response to infection (17). It can occur with or without bloodstream infection. The predictive validity of the qSOFA for in-hospital mortality outside of intensive care units was higher than that of the SOFA and SIRS criteria (which are more elaborate). However, as qSOFA was validated on patients already suspected of infection, it cannot be used to differentiate patients with infection from those without infection.

In Figure 3, we propose a set of clinical indications for sampling blood cultures in LMICs. These indications were based on known common causes of BSI (3), the qSOFA criteria (13) and experience within our research network $(18,19)$. For neonates, blood culture indications are different and more complex; we refer to other resources for more information (20-22).

\section{Collection of Blood for Blood Culture}

The process of collection of blood for blood culture is very important for the quality of the results (23). It requires applying a tourniquet on the arm, palpating the vein that will be used for the sampling, and applying appropriate antiseptics at the place of sampling. After antisepsis, the vein should not be touched anymore unless wearing sterile gloves. Next, the vein is pierced with either a needle and syringe or a butterfly needle. A sufficient volume of blood is aspirated either directly into the blood culture bottle (with certain bottle types and use of butterfly needle) or into a syringe and next divided over the blood culture bottles. 


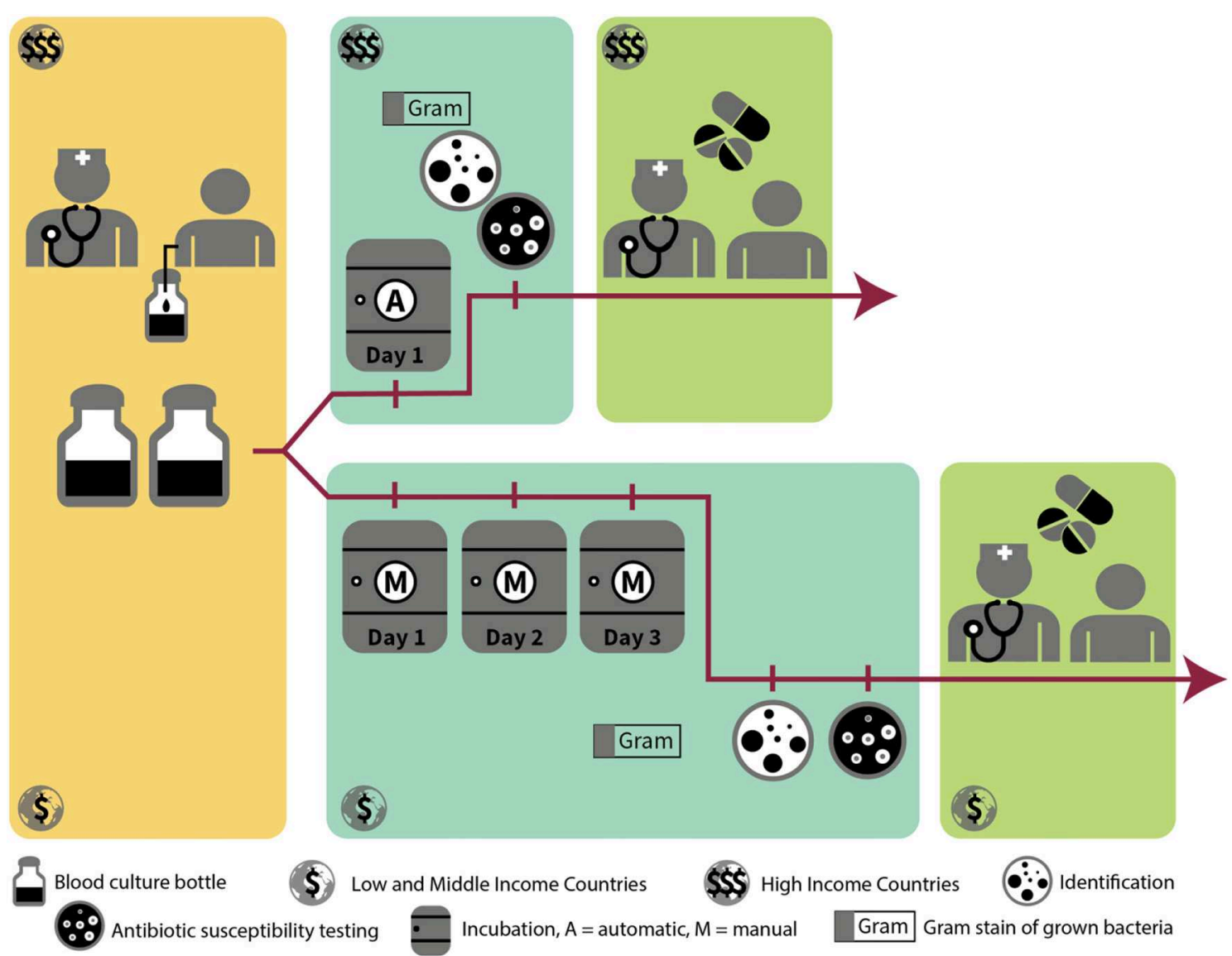

FIGURE 1 | Workflow of grown blood cultures in high-resource vs. low-resource setting.

\section{Automated vs. Manual Blood Cultures}

Further processing of cultures is described in this text with an emphasis on so-called "manual" blood culture systems (i.e., not making use of automated equipment). These systems rely on the use of appropriate blood culture bottles, which are placed in a conventional static incubator and are inspected daily to visually detect signs of growth of micro-organisms. This contrasts with the automated systems, which over the past decades have largely replaced manual blood culture systems in HICs and are the current standard (23). In these systems, the carbon dioxide production by micro-organisms in the bottles is continuously monitored, either by colorimetric or fluorescent detection. During incubation, the bottles are continuously agitated in the automated equipment. The current automated systems show better performance than manual systems in terms of yield and especially speed of growth, as recent studies from LMICs such as Egypt, Pakistan, and India have shown (24-26). However, these automated systems are costly, require regular maintenance and are not adapted to tropical, dusty environments, impeding the sustainable implementation of this technique in many LMICs outside of study sites, large reference laboratories or private laboratories in the capital cities $(1,27)$. Therefore, manual blood culture bottles are still most frequently used in LMICs and contribute worldwide to approximately the double of market share compared to automated systems (27). These manual blood culture bottles will be discussed and evaluated keeping the World Health Organization's (WHO) ASSURED criteria for diagnostics in LMICs in mind; i.e., they must be affordable, sensitive, specific, user-friendly, robust, rapid, equipment-free, and delivered to those who need it (28). Recently, the same research group proposed to add two criteria to this list: the REASSURED criteria put an additional emphasis on real-time connectivity and ease of specimen collection (29).

\section{The Importance of Blood Cultures in LMICs}

BSI presents a high burden of morbidity and mortality worldwide, with the highest burden in neonates and children (30). Exact figures for incidence and associated mortality of BSI are scarce to non-existent in many LMICs, because of a lack of bacteriological laboratories and surveillance (31-34). Even in HICs, however, mortality of BSI is still substantial, ranging between 17 and $29 \%(31,32,35,36)$. Key interventions to decrease mortality of BSI are sampling of blood cultures before administration of antimicrobial therapy and daily reassessment of antimicrobial therapy for optimization and de-escalation, based on the identification and antibiotic susceptibility testing of the pathogen (37). As survival of BSIs is inversely related with 
TABLE 1 | Examples of common bacterial species grown in blood cultures.

\begin{tabular}{|c|c|c|c|c|c|}
\hline & \multicolumn{2}{|c|}{ Gram-positive } & \multicolumn{2}{|c|}{ Gram-negative } & \multirow{2}{*}{$\begin{array}{l}\text { Yeast } \\
\text { Pathogen }\end{array}$} \\
\hline & Pathogen & Contaminant & Pathogen & Contaminant & \\
\hline \multirow[t]{3}{*}{ Aerobic } & & Bacillus species & Pseudomonas aeruginosa & Stenotrophomonas maltophilia* & Cryptococcus neoformans \\
\hline & & & Burkholderia pseudomallei & Pseudomonas species (non-aeruginosa) & \\
\hline & & & Acinetobacter species & & \\
\hline Anaerobic & Clostridium species & Cutibacterium acnes & Bacteroides species & & \\
\hline \multirow[t]{4}{*}{$\begin{array}{l}\text { Facultative } \\
\text { /aero-tolerant }\end{array}$} & $\begin{array}{l}\text { Streptococcus } \\
\text { pneumoniae }\end{array}$ & $\begin{array}{l}\text { Coagulase-negative } \\
\text { Staphylococcus spp. }\end{array}$ & Escherichia coli & & Candida albicans \\
\hline & $\begin{array}{l}\text { Staphylococcus } \\
\text { aureus }\end{array}$ & Micrococcus species & Klebsiella pneumoniae & & Candida glabrata \\
\hline & & & Non-typhoidal Salmonella & & \\
\hline & & & Salmonella Typhi & & \\
\hline
\end{tabular}

*Uncertainty of interpretation according to current literature.

time to adequate antibiotic therapy (38), it is also important that results of blood cultures are available as soon as possible.

Historically, the default acute fever diagnosis in tropical settings has been malaria, which still represents a major childhood killer. Based on symptoms and clinical presentation, severe malaria is indistinguishable from BSI, contributing to overdiagnosis of malaria and underdiagnosis of BSI particularly in children $<5$ years old $(39,40)$. Moreover, morbidity and mortality of malaria have been declining steadily over the last few decades. The number of malaria deaths globally fell from an estimated 839,000 in 2000 , to 445,000 in 2016 , which is a decline of $47 \%$ (WHO World Malaria Report $2017^{2}$. In contrast, progress in outcomes for sepsis has been much more modest, with a decline in mortality from sepsis of only $25 \%$ between 2000 and 2015-moving it upwards in the rank of deadly diseases requiring pathogen-based diagnosis (41). Increasing evidence of co-infection of malaria with non-typhoidal Salmonella (42, 43) further emphasizes the importance of blood culture in these settings.

The spectrum and frequency ranking of bacteria causing BSI in LMICs differ from those recorded in HICs. Pathogens like Salmonella enterica or Burkholderia pseudomallei are uncommon in HICs but account for a large proportion of pathogens in Africa and South-East Asia (5, 19, 44-47). Typical childhood pathogens for which vaccination is available in HICs, such as Haemophilus influenzae and Streptococcus pneumoniae, are also more common in LMICs $(45,46)$.

\section{Feasibility of Blood Cultures in LMIC Settings}

In the majority of blood cultures only one organism grows. Polymicrobial infections make up $6-18 \%$ of all BSI episodes, with higher proportions of polymicrobial infections seen in patients with chronic conditions, malignancies and nosocomial infections (48-54). Polymicrobial infections are probably less prevalent in LMICs, because of differences in patient population. A 2010 review of BSI in Africa found only 1.2\% of BSI

${ }^{2}$ https://www.who.int/gho/malaria/epidemic/deaths/en/ episodes to be polymicrobial (45). An analysis of BSI episodes in Cambodia showed that only $4.9 \%$ of episodes were polymicrobial (5). A convenient consequence of this is that the work-up of blood cultures for identification and antibiotic susceptibility testing is relatively straightforward, as no further isolation of different possible pathogens has to be done. In comparison to other specimens, it is usually easy to differentiate between pathogens and contaminants, and correct identification of the most common causative pathogens can be achieved with simplified techniques. Indications for blood cultures are simple to standardize hospital-wide, and quality indicators to monitor compliance to procedures are more clearly defined than for other specimens (1).

For these reasons, blood culture is an easy first step in starting clinical bacteriology in any laboratory, and it is recognized as a priority specimen for surveillance of antimicrobial resistance by WHO because of its clinical importance and the accurate and uncomplicated methods of detection (55).

\section{Importance of Blood Culture for Antibiotic Stewardship and Hospital Infection Prevention and Control}

Because of the severity of most BSIs, blood cultures have high clinical relevance. Rapid notification of even preliminary blood culture results, such as the result of a Gram stain of a grown culture, has been shown to have a large impact on rational antibiotic prescriptions, length of hospitalization and even patient survival (56-62).

Moreover, results from blood culture surveillance can be efficiently used in infection prevention and control. Analysis of trends per hospital ward can alert outbreaks $(63,64)$. Moreover, blood culture results allow to make the distinction between healthcare-associated and community-acquired infections. The WHO defines a healthcare-associated infection as "an infection occurring in a patient during the process of care in a hospital or other health-care facility which was not present or incubating at the time of admission" (65). This term has replaced the term "nosocomial" or "hospital-acquired" infection, as it was acknowledged that the epidemiological and microbiological 


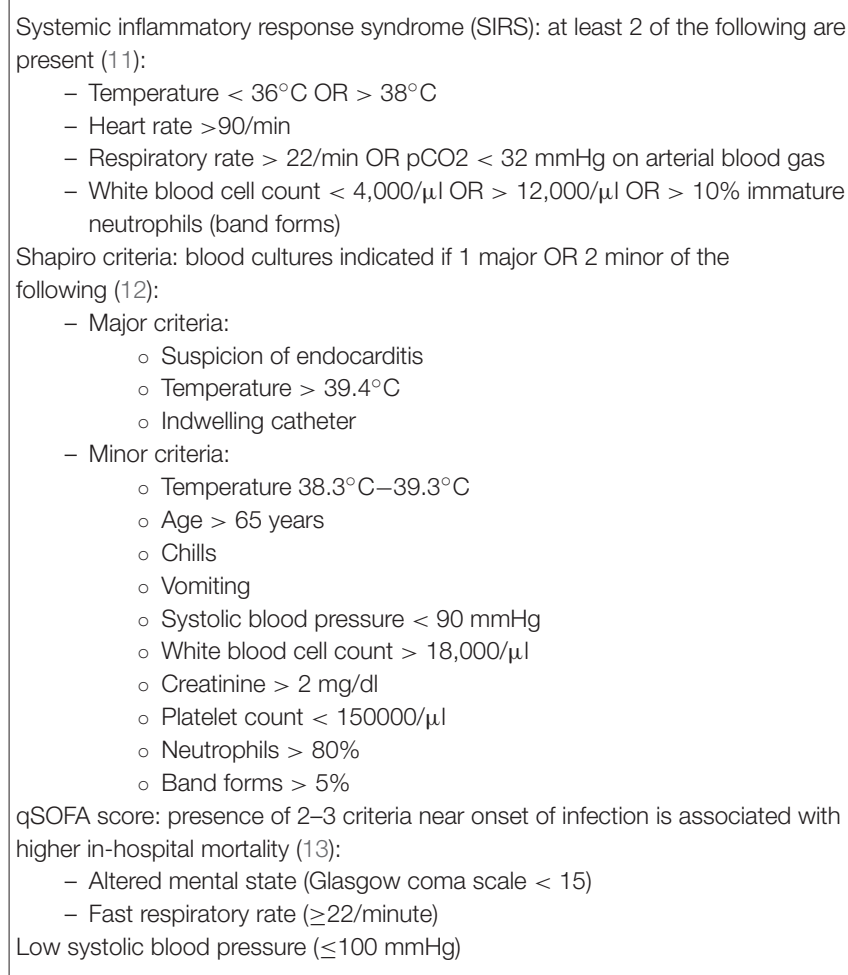

FIGURE 2 | Models and scores to predict BSI: SIRS criteria, Shapiro criteria \& qSOFA score (11-13).

features of infections acquired in the hospital were very similar to those acquired in other types of healthcare facilities (65-68). A healthcare-associated infection is thus defined as an infection that presents itself more than $48 \mathrm{~h}$ after the patient has been admitted to the hospital, or within $48 \mathrm{~h}$ of admission if the patient received care at any other facility (including at home) before the hospital admission (66).

However, specialized home care and referrals from other healthcare facilities are rare in LMICs and usually healthcareassociated infections in these settings can accurately be defined as infections with a first positive culture obtained at least $48 \mathrm{~h}$ after the moment of admission (similar to the previous definition for hospital-acquired infections). This definition is also used in the Global Antimicrobial Resistance Surveillance System (GLASS) report for use in surveillance of healthcare-associated infections (55).

Monitoring and surveillance of blood culture results can shed a light on the type, impact and number of healthcare-associated infections in a facility. This information can guide selection of appropriate prevention and control measures.

\section{METHODS AND TECHNIQUES FOR PROCESSING MANUAL BLOOD CULTURES}

Despite continuous advances in molecular techniques and biomarkers, blood culture remains the gold standard for
1. Fever (axillary $\mathrm{T}^{\circ} \geq 38^{\circ} \mathrm{C}$ ) OR history of fever (last $48 \mathrm{~h}$ ) OR hypothermia (axillary $\mathrm{T}^{\circ} \leq 36^{\circ} \mathrm{C}$ )

2. AND one of the following signs of severity:

A. Hypotension (systolic blood pressure $=<100 \mathrm{mmHg}$ )

B. Confusion (Glasgow coma scale $<15$ )

C. Increased respiratory rate ( $>=22$ per minute)

D. Suspicion of severe localized infection:

- Pneumonia

- Meningitis

- Osteomyelitis

- Complicated urinary tract infection

- Abscess

- Skin or soft tissue infection

- Abdominal infection

E. Suspicion of other severe infection:

- Severe malaria

- Typhoid fever

- Endocarditis

FIGURE 3 | Proposal for clinical indications of bloodstream infections in LMICs $(3,17-19)$.

diagnosis of BSI (23). As mentioned above, LMICs still largely rely on manual blood culture systems, because of the financial and logistic challenges associated with automated systems.

Manual blood cultures are usually incubated for 7 days at $35^{\circ} \mathrm{C}$ $(69,70)$. The incremental value of bacterial growth on day 6 and 7 may be limited; a study from 1985 showed that $89 \%$ of isolates had been recovered by day 5 ; many of the isolates retrieved on day 6 and 7 were probable contaminants (71). This observation holds true even for fastidious organisms, such as the HACEK group bacteria (see Table 1 for definitions) (72). As local differences in pathogens can impact the effect of a shorter incubation, the guidelines of the American Society for Microbiology (ASM) recommended examining the impact of a 5-day instead of a 7day incubation per site and described a procedure to do so in its 2004 edition (73).

For automated blood culture systems, incubation of 5 days has been shown to be sufficient, even for fastidious organisms such as members of the HACEK group (endocarditis) and Brucella species $(72,74)$ (see Table 1). For most pathogens, incubation of 3 days would already suffice (75).

\section{Broth Type and Additives to Promote Growth in Blood Cultures \\ Broth Medium}

The most important feature of a blood culture bottle is that it should adequately support bacterial growth. For this purpose, a variety of peptide broths and additives are available (see Table 3 ). There is no single "optimal" broth medium; most broths contain dextrose and animal-derived complex peptide molecules. Broth media which support growth of a wide range of bacterial species include tryptic soy broth, also known as soybean-casein digest broth, supplemented peptone broth and brain heart infusion broth. Other commonly used broths such as thioglycolate broth, thiol broth, Columbia and Brucella (hypertonic) broth are also adequate for bacterial recovery $(69,88)$. For anaerobic bacteria, 
TABLE 2 | Definitions used in or related to the blood culture process.

\begin{tabular}{ll}
\hline $\begin{array}{l}\text { Automated blood culture } \\
\text { system }\end{array}$ & $\begin{array}{l}\text { Blood culture system that uses equipment (an } \\
\text { automated incubator) for incubation, agitation, and } \\
\text { monitoring of blood culture bottles for microbial } \\
\text { growth }\end{array}$ \\
Bacteremia & $\begin{array}{l}\text { Presence of bacteria in the bloodstream } \\
\text { Biphasic blood culture }\end{array}$ \\
system & $\begin{array}{l}\text { Blood culture system in which a single bottle } \\
\text { consists of a liquid broth phase and a solid agar } \\
\text { phase; designed so the agar can be irrigated (and } \\
\text { inoculated) with the broth medium }\end{array}$
\end{tabular}

Blind subculture Subculture performed from the blood culture bottle in the absence of visual signs of growth

Blood culture Specimen of blood sampled through 1 venipuncture (possibly divided into multiple blood culture bottles) for the culture of micro-organisms

Blood culture broth A liquid enrichment medium for the growth of bacteria used in the diagnosis of BSI. Usually contains peptides of animal origin and dextrose.

Bloodstream infection Infection with the presence of bacteria in the blood Bloodstream infection A BSI episode is defined as (1) the initial recovery of a episode

Contamination

Culture medium

Endocarditis

Fastidious organisms

HACEK organisms pathogen in a blood culture, (2) the recovery of a pathogen different from the initial pathogen $\geq 48 \mathrm{~h}$ after the recovery of the initial pathogen, or (3) the recovery of the same pathogen after at least a 14-day interval since the previous grown culture with this pathogen $(5,6)$

Growth of a micro-organisms in a blood culture that was introduced into the culture during blood culture collection or processing and that is not the cause of the suspected BSI

Substance used to facilitate growth of bacteria; can be solid (agar) or liquid (broth)

Infection of the inner layer of the heart, the endocardium, often involving the heart valves

Organisms that require special nutritional and incubation conditions for culture (e.g., addition of certain nutrients, incubation in carbon dioxide atmosphere)

A group of Gram-negative bacteria that are unusual causes of endocarditis; consists of the following species: Haemophilus parainfluenzae, Haemophilus aphrophilus, Actinobacillus actinomycetemcomitans, Cardiobacterium hominis, Eikenella corrodens, and Kingella kingae (7)

Manual blood culture bottle Blood culture bottle that is designed for use in a manual blood culture system, i.e., without using automated equipment

Manual blood culture system

Non-fermenting

Gram-negative organisms (non-fermenters)

Subculture Blood culture system that processes blood culture bottles without the use of automated equipment

Heterogenous group of Gram-negative bacilli that are aerobic and cannot metabolize carbohydrates through fermentation; mainly implicated in healthcare-associated infections and often resistant to many types of antibiotics

A secondary culture of bacteria made from material derived from another culture, such as the blood-broth mixture of a blood culture bottle or the colonies on an agar plate

Terminal subculture
A subculture done at the end of the incubation period of blood culture bottles that failed to show signs of growth, to confirm the absence of growth of micro-organisms
TABLE 3 | Media composition of manual blood culture bottles.

\begin{tabular}{ll}
\hline $\begin{array}{l}\text { Medium or } \\
\text { Component/Additives }\end{array}$ & Short description/Comment/References \\
\hline CULTURE MEDIUM & \\
\hline Thioglycolate & Favors growth of anaerobes (76) \\
Tryptic Soy broth (TSB) & $\begin{array}{l}\text { General purpose medium, favors Pseudomonas } \\
\text { species (77, 78) }\end{array}$ \\
Thiol broth & $\begin{array}{l}\text { Favors growth of Streptococcus species (78) } \\
\text { Brain-Heart Infusion (BHI) }\end{array}$ \\
$\begin{array}{l}\text { General purpose medium, facilitates recovery of } \\
\text { yeasts and Gram-positive organisms (79) } \\
\text { General purpose medium, favors growth of anaerobes } \\
\text { Supplemented peptone } \\
\text { broth }\end{array}$ & $\begin{array}{l}\text { General purpose medium; superior to TSB for most } \\
\text { common pathogens in blood culture (80) } \\
\text { Hypertonic medium } \\
\text { (Brucella broth) }\end{array}$ \\
$\begin{array}{l}\text { Supposedly improves cellular stability and increases } \\
\text { recovery rates of some bacteria, including } \\
\text { Staphylococcus aureus, Escherichia coli, Candida } \\
\text { species (81); evidence regarding its efficacy is mixed } \\
\text { (77) }\end{array}$
\end{tabular}

ADDITIVES

Sodium-polyanethole Anticoagulant; SPS also inhibits lysozyme, inactivates sulfate (SPS) clinically achievable concentrations of some aminoglycoside and polymyxin antibiotics, inhibits parts of the complement cascade, and inhibits phagocytosis (69). Higher SPS concentrations, while promoting the recovery of Gram-positive cocci, decrease the recovery of Gram-negative bacteria. Certain species of bacteria are inhibited by SPS, such as Neisseria species, Peptostreptococcus anaerobius, Moraxella catarrhalis, and Gardnerella vaginalis $(69,77)$.

Gelatin Counteracts the inhibition of growth of bacterial species by SPS in-vitro $(80,82)$. Evidence for its clinical efficacy however is not strong (82-86).

Yeast extract Promotes bacterial growth (77)

Saponin Lytic agent (used in lysis-centrifugation system); improves recovery of Streptococcus species

Hemin (X-factor) Promotes growth of fastidious organisms such as Haemophilus influenzae and Neisseria species (87)

NAD (V-factor) Promotes growth of fastidious organisms such as Haemophilus influenzae and Neisseria species (87)

Pyridoxine Promotes growth of pyridoxine-dependent organisms such as certain Streptococcus species

Para-amino benzoic acid Inhibits the effect of sulfonamide antibiotics

Cysteine

Reducing agent; improves recovery of anaerobic bacteria and Streptococcus pneumoniae (77)

Columbia broth, pre-reduced peptone broths, thioglycolate broths, and thiol broths are advantageous, supposedly based on their low redox potential $(76,77,89-91)$.

Regarding the volume of broth, a blood-broth ratio of 1:5 to 1:10 should be respected to optimize growth (92). In children, higher blood-broth ratios (e.g., 1:50 to 1:100) are acceptable (93).

\section{Additives to Promote Growth}

Supplementation of the broth medium can further promote growth. A number of additives have been defined as growth enhancers in blood cultures, but the effect of additives is limited to the organism targeted for growth (see Table 3). 
One of the most important additives is sodium polyanethole sulfonate (SPS). This is an anticoagulant, which in addition has been shown to have a stabilizing effect on microbial growth in blood cultures $(69,88)$. The typical concentration of SPS ranges from 0.025 to $0.05 \%(3,77,88,94)$. The utilization of SPS has greatly reduced time-to-detection for many bacteria, and it is generally agreed to be an indispensable component of blood cultures.

Another commonly used additive is saponin, which is added in some blood culture systems as a lytic agent with subsequent centrifugation (Isolator system from Abbott Laboratories, Chicago, USA). A 1998 study also showed an increased recovery rate with shorter time-to-detection with the addition of saponin to the blood culture media of an automated system (95). Saponin is also widely used in anaerobic bottles in the BACTEC automated blood culture system, in combination with resinsupplemented aerobic bottles $(96)^{3}$.

\section{Resins and Charcoal}

All guidelines on sepsis management stress the importance of sampling blood for blood culture before administration of antibiotics (37). The negative impact of antibiotic use prior to sampling on the yield of blood cultures is substantial, as the presence of antibiotics in the serum can inhibit the growth of bacteria (97-100). However, patients in LMICs are often already under antibiotic treatment before presenting to the hospital (101), since antibiotics are often readily available over-thecounter in these settings (102-104). To counteract the effect of antibiotics on growth of bacteria in the broth, antibiotic removal devices have been in use since 1982 (105). Nowadays, this role is largely taken up by resins.

Antimicrobial-binding resins and charcoal are generally considered effective in increasing microbial recovery rates. More microorganisms, particularly staphylococci and yeasts, are recovered from formulations with these additives, compared to non-supplemented formulations (106-109). There is no firm clinical evidence that the increased microbial recovery rates are due to inhibition or removal of antibiotic substances, although invitro research has extensively shown this to be the case $(69,110-$ 113). Most resins are formulated as highly porous polyvinyl and benzene within a spherical bead; there are cationic ion-exchange resins and polymeric adsorbent resins (77). In addition to binding of antimicrobial agents, resin beads provide additional surface area for bacterial growth and help filter and bind components of the complement cascade (69). The exact formulations and balance between ratios of nutrients and resins is often proprietary and thus unknown.

The drawbacks of adding resins to a blood culture medium are the non-specific neutralizing and binding properties of the resins that can result in the removal of nutrients and other substances required for bacterial growth. Moreover, addition of resins to the blood culture broth changes the medium's dynamics, shortens its shelf life and impacts the visual reading of growth in the bottles (90). The addition of resins may even increase

$\overline{{ }^{3} \text { https://www.bd.com/resource.aspx?IDX }=11265}$ the time-to-detection for some bacteria, such as Pseudomonas species (113).

However, in automated systems, resin-supplemented broths are superior to general broths and broths containing charcoal (114-118). Importantly, resins also do not interfere as much with Gram staining and reading as does charcoal (114).

\section{Gas Phase of the Bottle}

For aerobic blood culture bottles, the headspace contains ambient atmosphere to which different amounts (5-10\%) of carbon dioxide $\left(\mathrm{CO}_{2}\right)$ have been added. For anaerobic blood culture bottles, headspace contains $\mathrm{CO}_{2}$ and nitrogen dioxide $\left(\mathrm{NO}_{2}\right)$. Actual amounts of $\mathrm{CO}_{2}, \mathrm{O}_{2}$ and redox potential vary widely, mostly depending on the manufacturing practices of the blood culture bottles (90).

A portion of the headspace atmosphere is evacuated to create a partial vacuum; thus, blood culture bottles contain an atmosphere in the bottle headspace that has a lower pressure than the atmosphere, enabling easy sampling (88). Not all commercially available bottles contain a vacuum; depending on the sampling techniques, this can create problems during sampling.

\section{Detection of Mycobacterial and Fungal Infections}

For detection of mycobacterial, fungal or yeast infections in the blood, other broth types and incubation times may be needed. For detection of yeast, such as Candida species, regular blood culture bottles and incubation times are advised (74), although Candida has a slightly longer time-to-detection than most bacteria $(119,120)$. Specialized bottles for detection of fungi in blood exist and are more effective than regular aerobic blood culture bottles (121-123), but are not routinely used.

In case of suspicion of invasive filamentous or dimorphic fungi infection, such as Histoplasma spp., the use of lysiscentrifugator tubes (Isolator system, Abbott Laboratories) is advised $(74,124)$. In this method, blood cells are lysed by saponin and the sample is centrifuged. The resulting sediment is then cultured directly on blood agar (69). Lysis-centrifugation can also be used for detection of bacteria, for which a study from India showed lower sensitivity but shorter time-to-detection than conventional blood culture using trypticase soy broth (125). Older studies had already shown good results for detection of bacterial or fungal BSI, but with the drawback of higher contamination rates $(126,127)$. Moreover, it is more timeconsuming than conventional blood culture.

For mycobacteria, lysis-centrifugation has also been used to good effect, including in some LMICs $(128,129)$. Currently, the use of specific bottles for mycobacteria is advised (74). Longer incubation times are necessary for both mycobacterial infections and filamentous fungal infections (74).

\section{Visual Detection \\ Visual Signs of Growth}

Growth detection of manual blood culture bottles depends on visible changes in the broth such as turbidity, hemolysis, puff balls, and gas production (see Figure 4). Inspection of the blood culture bottles is done by the laboratory technicians with 
the naked eye. Therefore, the blood culture bottle must be designed to optimize this visual detection. For example, seethrough plastic or glass must be used. The addition of resins or charcoal, described above, can create a haziness in the broth, compromising visual detection of growth (90), while addition of charcoal renders visual detection of growth nearly impossible due to the dark coloration of the broth.

\section{Biphasic Bottles}

Biphasic bottles can be an option to facilitate growth detection. They consist of a liquid phase (the broth) and a solid phase in the form of an agar slant (see Figure 5). These bottles were first developed by Castañeda in 1947 for the isolation of Brucella (130). Brucella species grow slowly with a need for extended incubation times. Because of hemolysis and debris of dying red blood cells, turbidity can no longer be reliably assessed beyond 7 days and repeated subcultures are needed to detect growth. By obviating the need for frequent subculture and its associated infection risks for the laboratory staff handling the blood cultures, biphasic bottles are useful for the isolation of this pathogen. Biphasic media also facilitate the detection of growth of other bacteria for non-expert users, as growth of colonies on the agar may be easier to detect than subtle changes in the broth. Additionally, there is the theoretical advantage of bypassing the initial subculture step in the process of identification and antibiotic susceptibility testing when using the colonies growing on the agar slant, resulting in a potential $18-24 \mathrm{~h}$ reduction of the turnaround time in comparison with the conventional blood culture bottles (131).

Published literature on the effectiveness of biphasic blood culture bottles for bacteria other than Brucella is scarce and there are no recent studies (131-136). Overall, those studies indicate superior performance of biphasic bottles for Gram-positives in comparison to other, non-biphasic, manual blood culture bottles. With regards to the speed of growth, a study by Brook et al. showed slower growth in the biphasic bottle as compared to a monophasic bottle (135). Weckbach et al. used a special biphasic bottle design (Figure 6A), physically separating the agar from the broth, and found faster recovery of yeasts (Candida spp.) and Pseudomonas spp. with the biphasic bottle compared to the conventional bottle. Because of easy subculture, isolated colonies were also available much sooner with the biphasic bottle compared to subculture from the regular bottle (131). Some other studies also found that detection of growth on the agar slant preceded detection of growth in the broth $(134,137)$. However, the few studies done suffer from sample sizes and a lack of comparability which precludes sound conclusions on the advantages of the biphasic blood culture bottles.

A variation to this type of biphasic blood culture bottle is the connection of a cylindrical "paddle," containing solid agar plates, to a blood culture bottle, effectively separating the agar and broth phase (Figure 6B). Subculturing can then be done by inverting the bottle and flooding the agar plates with the broth-blood mixture. This can be done at various intervals to optimize isolation. A well-known example of this type of bottle was the Septi-Chek blood culture system by Becton-Dickinson Diagnostics (Franklin Lakes, New Jersey, United States), which was based on a conventional blood culture bottle to which a transparent plastic chamber was connected, which contained a panel of three solid agar media allowing to visualize growth and preliminary identification. This system had promising results and was widely used, but it is no longer commercially available (138-141).

\section{Volume of Blood to Be Sampled Low Concentration of Bacteria in Blood Necessitates Large Volume}

The concentration of micro-organisms in blood of patients with BSI ranges between $<1$ colony forming unit $(\mathrm{CFU}) / \mathrm{ml}$ to 10 $\mathrm{CFU} / \mathrm{ml}$ of blood in adults (4). To avoid missing BSI with a low bacterial load in the process of culturing blood, it is recommended to sample as large a volume as possible for culture. Multiple studies have already shown that higher volumes of cultured blood lead to higher rates of detection $(6,142-147)$. Therefore, the volume of sampled blood is an important quality indicator for blood cultures and should be monitored (3, 148, 149). Blood volume inoculated in a blood culture bottle can be estimated by weighing the bottle before and after sampling, translating the weight to volume by correcting for the density of blood $(1.06 \mathrm{~g} / \mathrm{ml})(150)$.

However, sampling large volumes of blood is not without risk of iatrogenic anemia in children (151). Moreover, it is not culturally acceptable to patients and healthcare workers in many LMICs $(152,153)$. Although this has not been widely studied, it is telling that even large clinical studies conducted in Africa using blood cultures sample only one bottle of 5-10 $\mathrm{ml}$ of blood in adults $(154,155)$.

\section{Recommendations for Adults}

For adults, both the Clinical Laboratories Standards Institute (CLSI) and Cumitech recommend to sample two blood cultures of $20-30 \mathrm{ml}$ of blood each $(3,88)$ over $24 \mathrm{~h}$, thus adding up to sampled volume of $40-60 \mathrm{ml}$. One blood culture is defined in these, and most other, guidelines as the volume of blood sampled through one venipuncture $(70,88)$. These recommendations are based on two older studies using manual blood cultures, where two consecutive blood cultures were found to detect 88 and $>99 \%$ of BSI episodes $(106,156)$.

More recent studies suggest that even higher volumes of blood should be cultured. Cockerill et al. found that with an automated blood culture system, $65.1 \%$ of BSI episodes were detected with the first blood culture (consisting of $20 \mathrm{ml}$ of blood) (6). By adding a second blood culture, $80.4 \%$ of episodes were detected, and up to $95.7 \%$ were detected when three blood cultures were used. A study by Lee in 2007 indicated similarly that two blood cultures of $20 \mathrm{ml}$ each in a 24 -h period detected $89.7 \%$ of BSI (147). In this study, three blood cultures of $20 \mathrm{ml}$ detected $98.2 \%$ of BSI and only with four blood cultures, $>99 \%$ of BSI episodes were detected. Another recent study confirmed the need for at least three blood cultures (or $60 \mathrm{ml}$ ) to be sampled, as they found that $7.5 \%$ of BSI episodes would have been missed without a third blood culture (157).

A particular situation in terms of number of cultures to sample is suspicion of endocarditis. In this case it is generally 


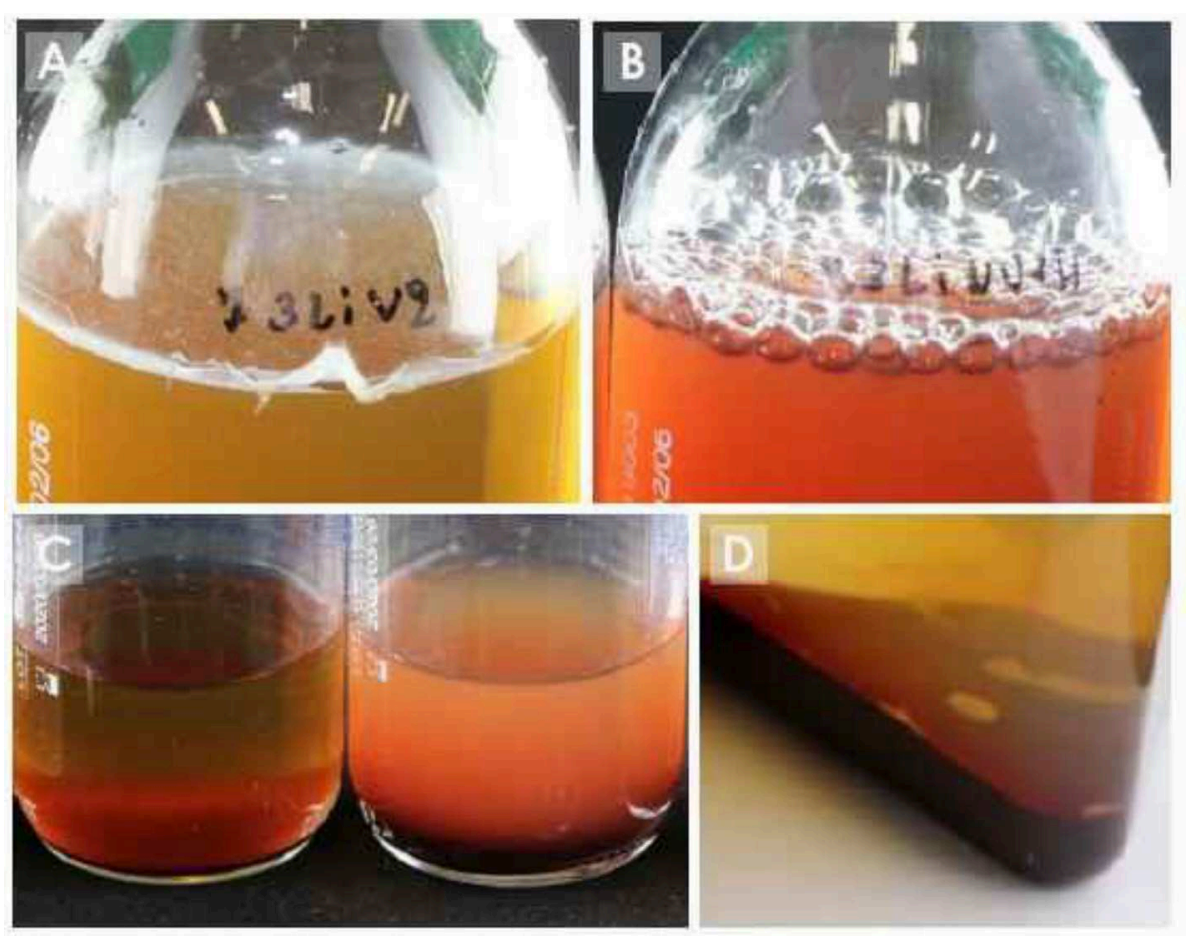

FIGURE 4 | Signs of growth in blood culture bottles. (A) pellicle formation on surface; (B) gas production; (C) turbidity (left bottle: no growth; right bottle: turbidity); (D) puff balls.

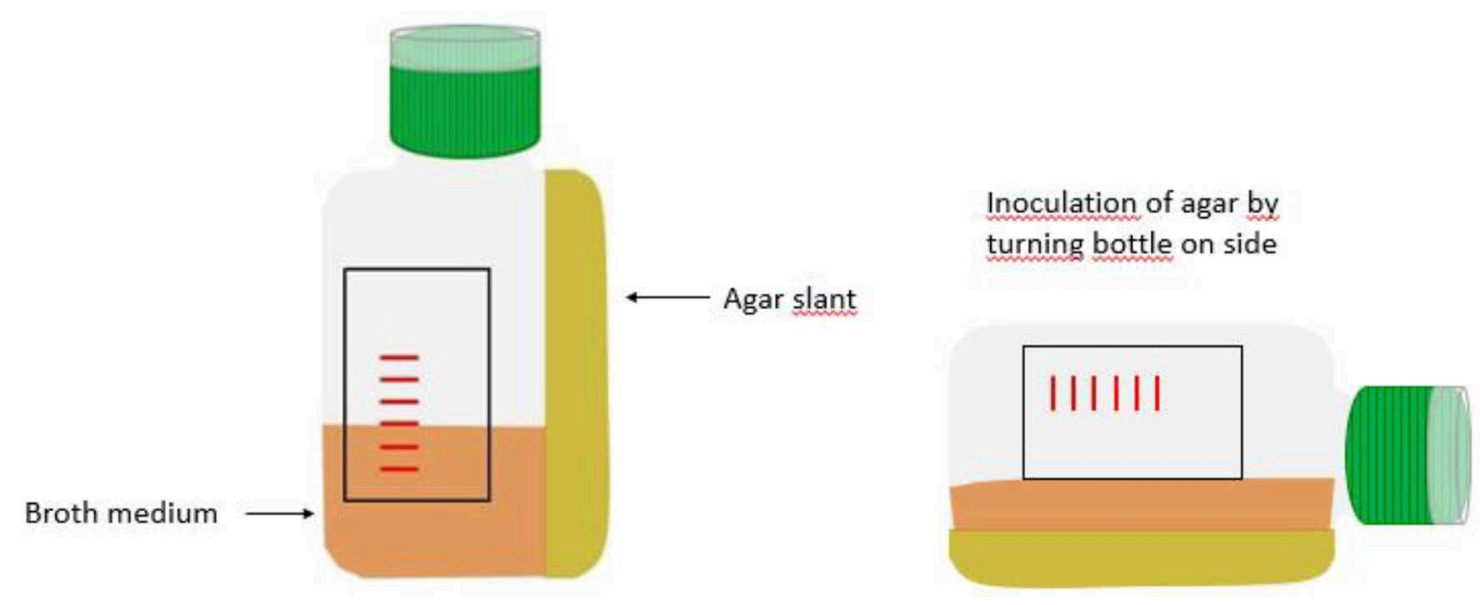

FIGURE 5 | Schematic representation of biphasic bottle.

recommended to sample three blood cultures of $20 \mathrm{ml}$, as this has showed to detect 96 to $98 \%$ of BSI in the context of endocarditis (158).

In many studies, a distinction is made between optimal volume per blood culture and optimal number of cultures to be sampled per BSI episode. However, as it has been established that drawing blood cultures at intervals or at the time of a fever peak does not contribute to a higher recovery of pathogens $(159,160)$, the key variable is the total volume of blood that is cultured, irrespective of time and frequency of sampling. For example, if it is recommended to sample $60 \mathrm{ml}$ of blood for culture, it may not make much difference whether this $60 \mathrm{ml}$ is cultured as two blood cultures of $30 \mathrm{ml}$ or as three blood cultures of $20 \mathrm{ml}(23,145,161)$.

\section{Recommendations for Children}

In children, the situation is more complex. The volume of blood that can safely and comfortably be sampled in children is related to the child's age and weight. It was long believed that very small volumes of blood were sufficient for blood culture in children, as bacterial concentrations in children with BSI were thought to be 


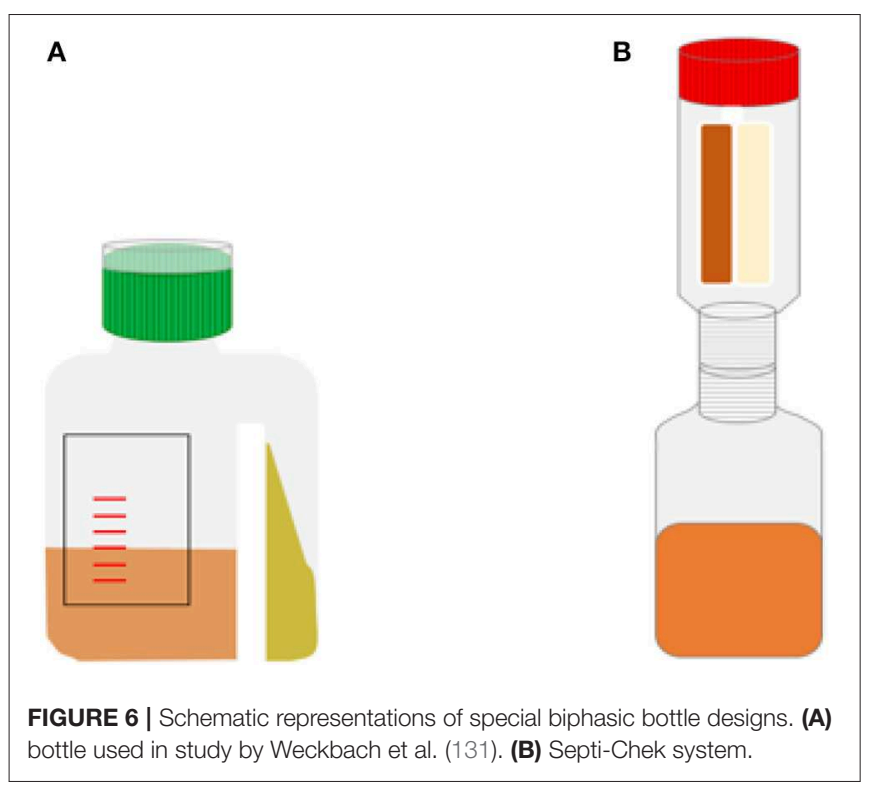

much higher than in adults (162). However, some studies have shown proportions of low-level bacteremia $(<10 \mathrm{CFU} / \mathrm{ml})$ in 23 to $69 \%$ of infants and children (163-165). In line with these, sampling higher volumes has been shown to increase sensitivity of blood cultures in children $(163,166,167)$. Given the high proportion of low-level bacteremia that was found by Kellogg et al. (163), the authors recommended to sample up to $4-4.5 \%$ of the patient's total blood volume.

The latest guidelines from the Infectious Disease Society of America and American Society of Microbiology, recommend sampling 2.5 to $4 \%$ of total blood volume from children (74) (Table 4). However, a review from 2011 suggests that only $3.8 \%$ of total blood volume can be safely sampled (for all laboratory analyses combined over a $24 \mathrm{~h}$ period) in children beyond the neonatal period, and this safety value is further challenged by higher rates of severe anemia in children in LMICs, particularly caused by severe Plasmodium falciparum malaria, which predisposes to Gram-negative BSI $(43,151,168,169)$. Given the necessity in many seriously ill children to sample blood for other analyses as well, sampling $4 \%$ of total blood volume for blood culture alone can therefore not be recommended in LMIC. More in line with these concerns, CLSI recommends sampling of maximum $1 \%$ of the total blood volume (for infants and younger children) (88).

Many articles however recommend an age-based sampling strategy illustrated in Table 5 (166, 170, 171). This age-based sampling is convenient and safe, as the volumes are noticeably smaller than with weight-based guidelines (see Figure 7 for an example in LMICs). However, no upper limits are defined for any of the age categories, leaving quite some room for interpretation. For children over 36 months of age, this guideline risks sampling insufficient volume. It is unlikely that the same volume is appropriate for a 4-year old child as for a 13-year old child, but this distinction is not made. Unfortunately, as CLSI remarks, "There are no published data for determining when volumes considered to be appropriate for adults can be used for older children" (88). As was pointed out by Dien Bard et al. in 2016, there is no consensus between different guidelines on which volume to sample in children (172), so controversy remains.

\section{Conclusions on Volume}

In conclusion, it is recommended that for adults at least $40 \mathrm{ml}$ of blood should be drawn, divided over 4 blood culture bottles, to obtain sufficient sensitivity. If feasible, it is recommended to sample an additional 20 to $40 \mathrm{ml}$ of blood. It is unnecessary to sample more than $80 \mathrm{ml}$ of blood, as $>99 \%$ of pathogens will be detected at this point (147). In case of suspicion of endocarditis, 3 blood cultures of $20 \mathrm{ml}$ each must be sampled. For children, recommendations are less straightforward. Because of simplicity, concerns regarding iatrogenic anemia, widespread use in hospitals and comparability across published literature, we recommend the use of the age-based simple algorithm depicted in Table 5 (e.g., sampling $\geq 0.5 \mathrm{ml}$ for neonates $<1$ month of age, $\geq 1 \mathrm{ml}$ for children age $2-36$ months and to sample $\geq 4 \mathrm{ml}$ in children $\geq 36$ months of age), despite our awareness of the limitations of this strategy.

\section{Preventing Contamination of Blood Cultures}

Sometimes, bacteria not present in the blood of the patient grow in blood cultures after having been introduced into the bottle during broth preparation, blood sampling or processing of the blood sample. These blood culture contaminants usually originate from the environment or the patient's skin (173). Most frequently, contamination happens during venipuncture, when skin fragments containing normal skin flora are dislodged and aspirated together with the sampled blood. Examples of common skin contaminants are coagulasenegative Staphylococcus species, Corynebacterium species and Cutibacterium acnes (formerly Propionibacterium acnes) (173). Contamination from the environment is also possible; Bacillus species (other than Bacillus anthracis) are often seen in this case.

\section{Distinction Between a Contaminant and a Pathogen}

It can be difficult to make the distinction between a contaminant and a pathogen, as some typical blood culture contaminants such as coagulase-negative Staphylococcus species can cause catheter infections or other foreign body infections. The distinction can be made either by clinical assessment (i.e., review of medical records), or by the number of blood cultures that show growth for this particular organism. Often, such an organism is only regarded as clinically relevant if it is isolated in at least 2 separate cultures (and venipunctures), because the odds of having contaminated both cultures with the same pathogen are very small (174). However, this approach cannot be used in settings where only one blood culture is sampled. Time-to-detection can also be of help in the interpretation, as it has been shown that contaminants show slower growth than true pathogens $(6,119,175)$.

Corynebacterium species, Bacillus species (other than Bacillus anthracis), Micrococcus species, Lactobacillus species and Cutibacterium species are rarely associated with clinical 
TABLE 4 | Recommended volumes of blood for culture in children.

\begin{tabular}{|c|c|c|c|c|c|}
\hline \multirow[t]{2}{*}{$\begin{array}{l}\text { Weight of } \\
\text { patient }(\mathbf{k g})\end{array}$} & \multirow[t]{2}{*}{$\begin{array}{l}\text { Total patient } \\
\text { volume }(\mathrm{ml})\end{array}$} & \multicolumn{2}{|c|}{$\begin{array}{l}\text { Recommended volume of blood for blood } \\
\text { culture }(\mathrm{ml})\end{array}$} & \multirow[t]{2}{*}{$\begin{array}{l}\text { Total volume for } \\
\text { culture (ml) }\end{array}$} & \multirow[t]{2}{*}{$\begin{array}{l}\text { Percentage of patient's } \\
\text { total blood volume }\end{array}$} \\
\hline & & Culture 1 & Culture 2 & & \\
\hline$\leq 1$ & 50-99 & 2 & - & 2 & 4 \\
\hline $1.1-2$ & $100-200$ & 2 & 2 & 4 & 4 \\
\hline $2.1-12.7$ & $>200$ & 4 & 2 & 6 & 3 \\
\hline $12.8-36.3$ & $>800$ & 10 & 10 & 20 & 2.5 \\
\hline$>36.3$ & $>2200$ & $20-30$ & $20-30$ & $40-60$ & $1.8-2.7$ \\
\hline
\end{tabular}

Based on IDSA Microbiology guideline (74).

TABLE 5 | Recommended blood volumes for blood culture in children based on age $(166,170,171)$.

\begin{tabular}{lc}
\hline Age & Volume of blood to be sampled \\
\hline$<1$ month & $\geq 0.5 \mathrm{ml}$ \\
$1-36$ months & $\geq 1 \mathrm{ml}$ \\
$\geq 36$ months & $\geq 4 \mathrm{ml}$
\end{tabular}

Girl of 18 months old with a weight of $10 \mathrm{~kg}$ and an hemoglobin value of $2 \mathrm{~g} / \mathrm{dL}$ (severe anemia):

Hemoglobin reference values at this age: $10.5-13.5 \mathrm{~g} / \mathrm{dl}$

Estimated total blood volume (TBVe): $10 \mathrm{~kg} \times 80 \mathrm{ml} / \mathrm{kg}=800 \mathrm{ml}$

Functional total blood volume (TBVf) (corrected for hemoglobin):

Worstcase $=\frac{800 \mathrm{ml}(=\mathrm{TBV}) \times 2 \mathrm{~g} / \mathrm{dL}(=\text { actual hemoglobin value })}{13.5 \mathrm{~g} / \mathrm{dl}(=\text { upper limit of hemoglobin reference value })}=118.5 \mathrm{ml}$

Bestcase $=\frac{800 \mathrm{ml}(=\mathrm{TBV}) \times 2 \mathrm{~g} / \mathrm{dL}(=\text { actualhemoglobinvalue })}{10.5 \mathrm{~g} / \mathrm{dl}(=\text { lowerlimitofhemoglobinreferencevalue })}=152.4 \mathrm{ml}$

Weight-based guideline IDSA : $6 \mathrm{ml}$ to be sampled for blood culture $=3.9 \%-$

$5 \%$ of TBVf

(see Table 4)

Age-based guideline : $\geq 1 \mathrm{ml}$ to be sampled (upper limit $=$ ?)

$1 \mathrm{ml}$ sampled for blood culture $=0.7 \%-0.8 \%$ of TBVf

$4 \mathrm{ml}$ sampled for blood culture $=2.6 \%-3.4 \%$ of TBVf

(see Table 5)

FIGURE 7 | Example of safe sampling in an 18-month old child with severe anemia caused by Plasmodium falciparum malaria [formulas used from Kuijpers et al. (169)]. The weight-based guideline recommends sampling of higher volumes than the age-based guideline. This example demonstrates how, in cases of severe anemia, the weight-based guideline may not be safe in children.

infections and are almost always contaminants (176). Coagulasenegative staphylococci can cause true infection but are much more often implicated as contaminants. Isolation of Enterococcus species, non-fermenting Gram-negative species (e.g., Acinetobacter species or Stenotrophomonas maltophilia) and viridans streptococci is often of uncertain clinical significance, complicating their role in the interpretation of blood culture results $(70,176)$.

\section{Contamination in LMIC}

Contamination of blood cultures remains a substantial problem, with contamination rates as high as $10 \%$ even in many HICs (177). Contamination is suspected to be more frequent in LMICs, with some settings (i.e., South Africa, Ghana, the Gambia, Malawi) indeed reporting very high contamination rates (178181). The spectrum of contaminants in LMICs differs slightly to HICs; for example, more Bacillus species are seen in LMICs (178). This finding suggests that contamination of blood cultures in LMICs commonly has the environment as a source, as Bacillus species are known to be present in dust and have been described in outbreaks of pseudo-bacteremia originating from the environment $(182,183)$. More confusingly, some organisms representing "true pathogens" in HICs, such as Pseudomonas aeruginosa or other non-fermenting Gram-negative organisms, are sometimes considered as contaminants in LMICs $(181,184)$.

Laboratory work-up of these contaminants demands time and money, both of which are not in large supply in resourcelimited settings. Furthermore, contaminants lead to longer hospital stays, increased prescription of antibiotics and related morbidity (185-188), and may hamper growth of pathogens. Therefore, avoiding contamination is of utmost importance and can lead to substantial cost savings and improvements in patient management. Current guidelines advocate a target of $<3 \%$ contamination rate and ideally $<1 \%(70,88,173)$. In the following paragraphs, some strategies are described to avoid contamination.

\section{Strategies to Reduce Contamination Skin antisepsis}

WHO's recommendation from 2010 regarding the type of antiseptic preferably used for blood sampling in the context of blood transfusion is $2 \%$ chlorhexidine in $70 \%$ isopropyl alcohol (189). No guidance is given regarding blood sampling in the context of blood culture. The CLSI guideline of 2007 on procedures for blood cultures states that tincture of iodine (alcohol-based iodine) and chlorhexidine gluconate are probably equivalent to each other but superior to povidone iodine (waterbased iodine) (88). Unlike iodine, chlorhexidine is not associated with allergic reactions, although its safety in infants $<2$ months of age has not been established (190-192). A lot of evidence suggests that products containing alcohol are better than those without alcohol (193-199), a finding which may be due to the fact that alcohol dries faster than water-based products and therefore less waiting time is required to obtain maximal disinfecting activity. Alcohol has an immediate onset of action, but the effect 
is not sustained, which is why it is often combined with other antiseptics with a residual effect, such as chlorhexidine (196).

In contrast to this, a recent meta-analysis found no significant difference between any of the antiseptics under study (povidone iodine, iodine tincture, chlorhexidine compounds, or chlorhexidine alcohol) (200), although its methods have been criticized (201). The debate on the most ideal antiseptic is thus ongoing, but most antiseptics seem to give good results when used properly. For this reason, alcohol-based antiseptics may be more effective, as the drying time is shorter, and they are therefore more likely to be used correctly. In addition, alcohol-based solutions are less vulnerable to accidental colonization with Gram-negative bacteria compared to waterbased antiseptics (202).

\section{One-step vs. two-step procedure}

The comparison of one-step (single application of an antiseptic) vs. two-step procedures (consecutive application of the same or different antiseptics) for skin antisepsis has been made mainly in the context of blood collection for blood donation. These studies generally evaluate the effect of antiseptics on the bacterial load of the skin (203). A Cochrane review of 2015, however, found no experimental or quasi-experimental publications which had studied the effect of one-step vs. two-step procedures on actual blood culture contamination (204).

Therefore, no recommendation can be given regarding the effectiveness of either procedure. In many LMICs, patients have had long travels on dusty roads before reaching the hospital. It is therefore good practice to use a two-step procedure for antisepsis: the first application of antiseptic (preferably isopropyl alcohol $70 \%$ ) can be used to clean the skin of dust and dirt. Cleaning with alcohol swabs must be repeated until the swabs are visually clean-since, unlike ethanol, isopropyl alcohol has a cleaning effect (205). Only when the skin is clean, the second step of antisepsis can be performed.

\section{Phlebotomy teams}

To further decrease contamination, there is quite good evidence for the use of dedicated phlebotomy teams for blood culture, as opposed to blood cultures being sampled by all ward nurses or interns $(193,197,206,207)$. This approach has also had good results in a low-income country; after the introduction of a team of nurses dedicated to phlebotomy in Malawi, contamination rates decreased from 19.6 to $5 \%$ (179).

\section{Blood culture collection packs}

Another possible strategy is the use of blood culture collection packs. Blood culture collection packs are prepackaged kits assembling all materials needed for blood culture. Although many studies have reported lower contamination rates after the introduction of blood culture collection packs (208-210), a metaanalysis of 2012 showed no conclusive evidence for this practice (207). Most of the publications which have reported a decrease in contamination rates combined introduction of the blood culture collection packs with hospital staff training and awareness campaigns, thereby compromising validity of the research. Given the added cost of blood culture collection packs, more evidence is needed before its implementation can be recommended in LMICs (207).

\section{Diversion of first portion of blood}

Contamination of blood samples can occur during venipuncture because of dislodging of skin fragments colonized with bacteria. Diverting or discarding the first few milliliters of blood can possibly decrease contamination due to this cause. The first study done to evaluate this method was the trial by Patton and Schmitt (211), which indeed showed decreased contamination after diversion of the first $\mathrm{ml}$ of blood into a sterile Vacutainer ${ }^{\circledR}$ tube (Becton-Dickinson Diagnostics, Franklin Lakes, NJ, USA). Contamination went down from 2.8 to $1.4 \%$. This concept was repeated by Binkhamis et al. (212) and Rupp et al. (213); both studies found significant results with a reduction in contamination rates of $3.4-2.4 \%$ and $1.78-0.22 \%$, respectively. In all three studies, pre-intervention contamination rates were already low. In LMICs with higher contamination rates, the same relative reduction could potentially lead to a large decrease in contaminated samples.

Usually, it is advised to sample first blood cultures and only next the tubes for other blood analyses. This recommendation is based on the observation that reflux from contaminated citrate- or EDTA tubes to the syringe may occur, with possible contamination of the blood culture sample $(177,214-$ 216). However, these reports of "pseudo-bacteremia" caused by contaminated blood collection tubes all date from the 1970's and 1980's and the current risk for this is unknown. Given the effectiveness of diverting the first portion of blood, contamination could actually be reduced by sampling first other blood collection tubes if a butterfly needle with vacutainer system is used. Sterilization of blood collection tubes may further prevent cross-contamination.

\section{Multi-sampling vs. single-sampling strategy}

The CLSI guideline recommends collection of two to three blood cultures, each consisting of two blood culture bottles sampled through one venipuncture, per episode (88). However, it has been shown that a time interval between the different draws is unnecessary $(159,160)$. Therefore, the necessity of sampling blood using separate venipunctures, referred to as multi-sampling, has been contested. There are obvious practical advantages to sampling blood by just one venipuncture, referred to as single-sampling (23). This single-sampling approach may also theoretically decrease the risk of contamination $(161,217)$. Unfortunately, few studies directly comparing both strategies are available. One trial in France showed increased positivity rate, improvement of overall performance (sensitivity and specificity) and better compliance to protocol of the multi-sampling strategy compared with the single-sampling strategy (218).

Contamination, however, can be more difficult to judge when single-sampling instead of multi-sampling is used. The reason for this is that many low-virulence organisms are no longer considered as contaminants when they are isolated from blood cultures sampled through more than one venipuncture $(69,88,174)$. However, an evaluation by Arendrup et al. did not find the interpretation to be more difficult when using the 
single-sampling strategy (219). They also demonstrated that the recovery of pathogens was correlated with the number of grown blood culture bottles, suggesting this indicator can be used to determine the presence of contaminants.

The recommended approach will probably depend on the setting and patient population; in a setting with very few central venous catheters or prosthetic devices, it is probably not needed to sample from different venipuncture sites, because infections with organisms of low virulence are very unlikely in this setting. In many LMICs there is a reluctance to sampling blood in both staff and patients (1), so a single-sampling strategy will probably more acceptable. In settings where the interpretation of possible contaminants is more difficult, it is still recommended to sample from at least two separate venipunctures.

\section{Sampling through catheters}

Many studies have shown increased contamination rates when blood is sampled through intravascular catheters, as opposed to venous puncture (177). However, most of this evidence is found when using central lines for blood sampling. This suggests that sampling blood when placing a peripheral intravascular catheter might be safe and practical, especially in children in whom a reduction of the number of venipunctures is desirable. Indeed, some studies did not show a higher contamination rate from blood sampled through a recently placed peripheral catheter than through separate venipuncture (220-223). However, many other studies have found higher contamination rates with this practice (224-227). Hall et al. described contamination rates as low as $1.6 \%$ when sampling via newly inserted catheters by following a sterile technique protocol in a pediatric emergency department (228). In general, sampling through peripheral catheters must be discouraged, but its practical advantages cannot be denied and should be balanced against the risk of increased contamination.

\section{Disinfecting the blood culture bottle septum}

Another measure to decrease contamination is to disinfect the blood culture bottle septum with $70 \%$ isopropyl alcohol or ethanol before injecting the blood sample $(88,193,229,230)$; in many institutes, however, this is not done routinely $(193,231)$. Bekeris et al. did not find evidence for the effectiveness of this measure in decreasing contamination, however they noted that their study was not powered to detect such an effect as they had quite low rates of contamination overall (206). In the context of higher environmental contamination in LMICs, disinfection of the blood culture bottle septum is an easy and low-cost strategy to avoid contamination.

\section{Sterile gloves}

Sterile gloves may reduce contamination rate but increase the cost of blood cultures $(232,233)$. Whether this incremental cost will be offset by decreased costs associated with work-up of contaminants will need to be evaluated and will depend on the baseline contamination rate (177). As there are many inexpensive ways to decrease contamination, sterile gloving might not be the most cost-effective option when looking for a strategy to reduce contamination rates. In most studies evaluating the use of sterile gloving, the absolute reductions in contamination rates following its implementation were low, leading to a high cost for very few averted contaminants $(232,233)$. However, baseline contamination rates were also low in these studies; in settings where contamination is much more prevalent, such as most LMICs, the same relative decrease in contamination rates will lead to a higher number of averted contaminants. In that case, the intervention will have a better cost-benefit ratio.

\section{Collection system}

Although no impact on contamination rates has been described to our knowledge, the choice of the type of collection system will be briefly discussed in this paragraph. For collection of blood cultures, either a butterfly needle with Vacutainer ${ }^{\circledR}$ system or a simple needle with syringe method can be used. It is not possible to increase the safety of the needle/syringe system by using safety devices (such as retractable needles), as the healthcare worker will still use the needle after sampling to inoculate the blood in the blood culture bottle. The time between sampling and inoculation into the bottle is the period of risk of needle stick injury (234). It is also not recommended to connect a blood culture bottle directly to the needle using a Vacutainer ${ }^{\circledR}$ system, as backflow of the broth into the patients' veins may occur. Butterfly needles on the other hand, are easy to use, especially when other blood analyses must be done as well; and other blood collection tubes can be connected to the Vacutainer ${ }^{\circledR}$ system after the blood culture bottles have been filled without the need for a separate venipuncture. Additionally, they are probably safer to use than needle-and-syringe as connection to the bottle is easier (provided the correct adaptor) (234). However, they are more expensive. If financially sustainable, butterfly needles are therefore recommended.

Lastly, changing needles between taking blood cultures and inoculating the blood into the blood culture bottle (in case of syringe and needle procedure) may decrease contamination rates slightly (235); however, it also increases the risk of needle stick injury and it is therefore not recommended $(88,189)$.

\section{Monitoring of Quality Indicators}

Unlike for other specimens, such as urine and respiratory secretions, many quality indicators which are useful to assess, and monitor have been described for blood cultures. They can be used to improve the processes from request to report in the hospital and laboratory by giving directed feedback to the staff involved. Examples of quality indicators are given in Table 6. Although many of these indicators seem clear-cut at first sight, definitions are not always uniform, and this leads to varying rates and figures being reported.

For example, contamination rate will vary across studies depending on how contamination has been defined and calculated, and often this is not well-described. According to Leber, the contamination rate is calculated "by dividing the number of cultures containing contaminants by the total number of cultures collected by venipuncture," with a blood culture being defined as "blood from a single venipuncture" (70). This suggests that blood culture contamination rates are defined as the contamination per venipuncture (and not per blood culture bottle), and it is thus important to know how the bottles were 
TABLE 6 | Quality indicators for monitoring of blood cultures - they can be used for validation as well as for monitoring purposes.

\begin{tabular}{|c|c|c|c|}
\hline Quality indicator & Definition & Goal & Comment \\
\hline $\begin{array}{l}\text { Proportion of blood cultures } \\
\text { that show growth with a } \\
\text { pathogen (positivity rate) }\end{array}$ & $\begin{array}{l}\text { Number of blood cultures showing growth } \\
\text { with a pathogen /total number of blood } \\
\text { cultures }\end{array}$ & $\begin{array}{l}5-15 \%(3) \\
6-12 \%(70) \\
\text { If lower than the goal, ordering of } \\
\text { blood cultures is too liberal; if higher } \\
\text { than the goal, it is too stringent }\end{array}$ & $\begin{array}{l}\text { These figures are appropriate for HICs and } \\
\text { settings where malaria is not endemic. } \\
\text { Studies performed in LMICs often show } \\
\text { higher proportions of pathogens (19). }\end{array}$ \\
\hline $\begin{array}{l}\text { Total number of blood } \\
\text { cultures }\end{array}$ & $\begin{array}{l}\text { Number of blood cultures/1000 patient } \\
\text { days }\end{array}$ & 103-188 (3) & $\begin{array}{l}\text { Has exclusively been studied in HICs; } \\
\text { goals for LMICs not clearly defined }\end{array}$ \\
\hline Missed opportunities & $\begin{array}{l}\text { Number of missed opportunities for blood } \\
\text { culture sampling as assessed by patient } \\
\text { file review (236) }\end{array}$ & Not defined & \\
\hline Contamination rate & $\begin{array}{l}\text { Number of contaminated blood } \\
\text { cultures/total number of blood cultures }\end{array}$ & $\begin{array}{l}<3 \%(3,70) \\
<1 \%(70)\end{array}$ & $\begin{array}{l}\text { For this definition, blood culture is defined } \\
\text { as blood sampled through one } \\
\text { venipuncture }\end{array}$ \\
\hline Volume & Volume per blood culture bottle & $\begin{array}{l}\geq 80 \% \text { of recommended volume } \\
(3,70,88)\end{array}$ & $\begin{array}{l}\text { Following formula can be used (the factor } \\
0.94 \text { expresses the correction for density } \\
\text { of blood): } \\
\text { Volume per bottle = (weight of bottle after } \\
\text { sampling - weight of bottle before } \\
\text { sampling + average weight of cap) * } 0.94\end{array}$ \\
\hline $\begin{array}{l}\text { Number and proportion of } \\
\text { solitary blood cultures }\end{array}$ & $\begin{array}{l}\text { Adult blood cultures consisting of only one } \\
\text { blood culture bottle instead of at least two }\end{array}$ & $\begin{array}{l}\text { Best performing hospitals have only } \\
3.4 \% \text { solitary blood cultures (3) }\end{array}$ & \\
\hline Needle-to-incubator time & $\begin{array}{l}\text { Time interval from blood culture sampling } \\
\text { to incubation }\end{array}$ & $\begin{array}{l}<2 \mathrm{~h}(88) \\
<4 \mathrm{~h}(70)\end{array}$ & \\
\hline Time-to-detection of growth & $\begin{array}{l}\text { Time interval from incubation to detection } \\
\text { of growth }\end{array}$ & $\begin{array}{l}\text { Not clearly defined for manual blood } \\
\text { culture bottles }\end{array}$ & \\
\hline Gram stain accuracy & $\begin{array}{l}\text { Correlation between smear result (Gram } \\
\text { stain) and culture result. }\end{array}$ & & \\
\hline Turnaround time & $\begin{array}{l}\text { Time interval from registration of the } \\
\text { sample in the laboratory to reporting of the } \\
\text { result to the clinician ( } 237 \text { ) }\end{array}$ & & \\
\hline $\begin{array}{l}\text { Quality of antibiotic } \\
\text { susceptibility testing (AST) } \\
\text { report }\end{array}$ & $\begin{array}{l}\text { Correct interpretation and reporting of raw } \\
\text { results }\end{array}$ & & \\
\hline
\end{tabular}

sampled exactly. Another problem with contamination rates is the definition of contamination itself, which may not be uniform across studies. Depending on the study, the species involved in contaminated blood cultures may differ, the number of cultures growing the contaminant may or may not be taken into account, or clinical review of the patient files may or may not have been done to determine true contamination. It is therefore important to assess the definition used in the study when comparing contamination rates.

\section{CHALLENGES}

Although we have argued in paragraph 1.3 that blood cultures are feasible in LMICs, some significant challenges remain. First and foremost, clinical bacteriology facilities in LMICs have to be strengthened and supported. As has been argued elsewhere, problems of staff training and retention, insufficient infrastructure, and lack of stringent quality control and equipment maintenance jeopardize all laboratory activities, including blood cultures (1, 2, 153). Other challenges, specific to blood cultures, will be discussed in the paragraphs below.

\section{Detection of Growth}

Manual blood culture systems require inspection of the blood culture bottles once or twice daily. Most signs of growth are rather subtle, and experience and training are therefore needed to recognize growth in the bottles. Manipulation of blood culture bottles can result in stirring the blood cells with the broth, which further complicates the detection of turbidity. Standardized visual conditions are preferable for reliable detection of growth. This includes standardized backgrounds and lighting; normal daylight may show substantial variation; therefore, the use of a lightbox can be considered.

\section{Blind Subcultures}

Because of the challenges related to visual detection of growth, other strategies were employed before the widespread use of automated monitoring of blood culture bottles. For manual systems, "blind subcultures" are often recommended. These are subcultures from the blood culture broth on agar plates, in the absence of visual signs of growth. The optimal timing of this subculture varies according to the consulted source. Depending on the moment of blind subculture during the incubation [for instance early at day 1 vs. at day 7 at the end of the incubation 
period (so-called "terminal" subculture)], blind subculture can be used either as a way to shorten the time-to-detection or as a final check for growth.

CLSI for example recommends subculture after 24 to $48 \mathrm{~h}$ of incubation to facilitate early detection of microorganisms (88). The American Society of Microbiology "Clinical Microbiology Procedures Handbook" by Leber et al. (70) recommends blind subculture at $72 \mathrm{~h}$. Cumitech advises routine blind subcultures after $12-18 \mathrm{~h}$ of incubation for aerobic bottles $(3,70)$. Routine blind subcultures are not advised for anaerobic bottles (3).

Early subculture (within the first $24 \mathrm{~h}$ of incubation) seems to be a successful strategy for rapid detection indeed. Szymczak et al. detected $85 \%$ of pathogens with blind subculture within $24 \mathrm{~h}$, whereas in only $48 \%$ of positive cultures visual signs of growth could be detected within $24 \mathrm{~h}$ of incubation (238). Even earlier subculture, within four to $14 \mathrm{~h}$ after incubation, led to detection of $50 \%$ (239) and up to $85 \%$ of pathogens (240). Studies performing subculture between 6 and $17 \mathrm{~h}$ after incubation detected 48\% (241) and 63\% (242) of pathogens. Subcultures performed within $6 \mathrm{~h}$ yielded only $10 \%$ of pathogens according to the study by Sliva et al. they therefore do not recommend subculture within $6 \mathrm{~h}$ of incubation (242). A drawback of blind subcultures is that by opening the bottle and introducing a syringe or pipette, contamination may be introduced.

\section{Terminal Subcultures}

Terminal subcultures (blind subcultures of blood culture bottles at the end of the incubation period) are considered unnecessary by many and may increase contamination rates and needle stick injuries (243-245). However, they may still be considered in systems which do not perform a routine blind subculture at an earlier time or in situations with prolonged incubation (245). They are also useful at the validation phase of a newly introduced blood culture system. In settings with many immunocompromised patients, where higher numbers of Pseudomonas aeruginosa and yeast BSI are anticipated, terminal subculture could also be of value, as these organisms tend to show slower growth and more subtle visual signs of growth, presumably due to smaller initial concentrations of bacteria (246).

\section{The Need for Speed: How to Decrease the Time-to-Detection and Turnaround Time}

It is well-known that automated blood cultures have a much shorter time-to-detection than manual systems. For manual systems, cumulative growth within $48 \mathrm{~h}$ of incubation varies between 65.8 and 94\% $(137,238,240,241,247,248)$. It must be noted that all of these studies performed a blind subculture within $24 \mathrm{~h}$ of incubation; not performing blind subculture may even lead to lower detection rates after $48 \mathrm{~h}$ of incubation. This is obviously longer than what is currently seen with automated systems, where $82-91.6 \%$ of growth is detected within $24 \mathrm{~h}$ (249-251). Some recent studies from LMICs, directly comparing manual with automated systems, also found higher sensitivity, specificity and especially much lower time-to-detection with the automated system (24-26).
The importance of reducing the time-to-detection of blood cultures cannot be overestimated, for both antibiotic stewardship purposes and to increase demand for blood cultures by clinicians in LMICs, as patients are often discharged or leave the hospital against medical advice early in the course of treatment $(252,253)$. Long turnaround times of test results are a common complaint in LMICs, decreasing the trust in the laboratory services $(254,255)$. Moreover, rapid communication of preliminary results, such as the Gram stain, is often not or insufficiently done in LMICs (153). Effective and immediate communication of blood culture preliminary results, ideally combined with clinical advice, should be a priority for bacteriology laboratories $(312,313)$.

\section{Agitation}

It may be impossible to increase the speed of detection of manual systems up to the performance of the automated systems, as the algorithm-driven continuous monitoring will prove difficult to mimic in an equipment-free manual system. However, another feature of automated systems is continuous agitation during incubation. Agitation is thought to improve microbial recovery by increasing the oxygen concentration in the broth medium and will therefore speed up detection of aerobic bacteria (69, 256-258). The "Clinical Microbiology Procedures Handbook" recommends continuous agitation of manual systems as well (70). Continuous agitation, however, impacts ease of visual growth detection, as turbidity will increase due to mixture with the red blood cells.

\section{Venting}

Another strategy to speed up growth is venting, i.e., inserting a filtered needle or other device that allows air to enter into the bottle headspace. The need for venting of aerobic blood culture bottle depends mainly on the manufacturer of the blood culture bottle. For automated systems, it is agreed that venting is not necessary $(69,259)$, but some commercial manual blood culture bottles may still need venting. Most studies evaluating the impact of venting stress the role of appropriate amounts of oxygen; this seems especially important for Pseudomonas, Candida and other strictly aerobic species, both for rate and speed of growth $(77,144,241,260)$.

It is currently unclear whether venting is still required with the newer commercial manual bottles; there are no recent studies on this. Of course, venting involves an extra procedure to be carried out, thereby potentially contaminating the sample. Despite this theoretical possibility, there is no documented evidence for increased contamination with vented bottles.

\section{Laboratory Organization}

Clinical importance of increased speed of detection is also influenced by laboratory organization. Notification of the Gram stain result to the clinician has been shown to have the highest clinical impact $(56,58,59)$. Laboratories not providing 24$\mathrm{h}$ service will probably see less benefit from small differences in time-to-detection, as they may be unable to translate this into quicker results to the clinician. Furthermore, the most important actionable result is growth in the first bottle of a BSI episode; growth in the other bottles of that episode are of lower 
clinical impact. When taking all this in consideration, the clinical relevance of difference in speed between automated and manual systems may be lower than expected. Future studies comparing speed of growth of different systems, including comparisons between automated and manual systems, should take these considerations into account.

A study that has looked at the practical organization in the lab was performed by Youngs et al. in 1985 (261). They recognized the added value of early subculture (after 6-17 h of incubation) but mentioned that the actual timing of subculture also depended on arrival of the bottle in the lab. To be sure that a subculture was performed within $6-17 \mathrm{~h}$, they implemented two timeslots for subculturing: one in the early morning for all bottles having arrived after office hours the previous day and another subculture at the end of the work day for all bottles that arrived during the day. This led to growth of pathogens on solid media the day after incubation for 10 out of 14 grown blood cultures, making an appreciable impact for patient management, as none of these bottles had shown visual signs of growth the day before (261).

\section{Transport to the Laboratory}

A relatively simple way of improving the speed of detection is by ensuring that the bottles arrive in the laboratory in a timely manner. It has been shown that a delayed incubation significantly prolongs detection time and may lead to falsenegative results in automated systems $(23,262,263)$, however the exact critical time has not been formally established. Guidelines recommend maximum needle-to-incubator time of $2 \mathrm{~h}$ (88) to $4 \mathrm{~h}$ (70); however, many centers actually observed much longer average transport times (264). Long distances and irregular transport of laboratory specimens from the site of collection to the laboratory is more common in LMICs, resulting in even higher transport times.

Blood culture bottles must be transported to the lab at room temperature $(88,265)$ or in a thermostable carrier and should never be refrigerated or frozen, as many fastidious organisms are vulnerable to cold $(88,266)$. Pre-incubation of blood culture bottles at $35^{\circ} \mathrm{C}$, for example in a small incubator in the emergency department, can speed up detection of growth in manual blood culture systems (267), but is not advised for automated systems as it may result in false-negative results $(23,268-270)$. However, high "room temperatures" are common in LMICs, warranting short transport times when using automated blood culture systems to avoid false-negative results.

\section{Direct Testing on Blood Culture Broth}

Performing direct identification and/or antibiotic susceptibility testing on grown blood culture broth can also lead to faster diagnosis and decision-making. Molecular assays and matrixassisted laser desorption/ionization-time-of-flight (MALDITOF) methods are currently out of reach for most LMICs, but lateral-flow immunoassays and other simple testing methods can provide a convenient solution. A number of simple direct tests have been described. Examples are detection of Staphylococcus aureus by direct tube coagulase test for detection of Staphylococcus aureus $(271,272)$, latex agglutination tests (273) and immunochromatographic tests (274), and the recent development of lateral flow assays for Burkholderia pseudomallei (275) and Salmonella species (276).

For antibiotic susceptibility testing, lateral flow assays have been developed to rapidly detect methicillin-resistant Staphylococcus aureus (277), extended-spectrum beta-lactamase (ESBL) (278) and carbapenemases (279), directly from blood culture broth. Moreover, the European Committee on Antibiotic Susceptibility Testing (EUCAST) recently provided guidelines on direct disk diffusion testing from blood culture broth, with adapted procedure and diameter cut-offs ${ }^{4}$. Performing antibiotic susceptibility testing directly from blood culture broth decreases the turnaround time with 1 day but requires rapid identification as the procedure often depends on the pathogen.

\section{Production, Distribution, and Procurement of Blood Culture Media \\ Accessibility and Affordability of Blood Culture}

Access to quality-assured in-vitro diagnostics is a problem in LMICs; diagnostics are frequently more expensive when purchased in a LMIC and they are not always easily available $(104,280,281)$. Although data are rare, it is estimated that the cost of blood cultures is twice as high in LMICs than HICs (41). Most healthcare systems in LMICs rely heavily on out-of-pocket payments of patients, including for diagnostics (153, 282, 283). According to a recent WHO report, out-of-pocket payments, although in decline, still account for $37 \%$ of the total healthcare cost in Africa and 44\% in South-East Asia (282). Assuming a positivity rate of only $10 \%$ for blood cultures, the cost-benefit ratio for the individual patient of performing blood culture may be unacceptable in very poor populations. A Kenyan study of 2009 reports a blood culture cost of 18.36 US \$ per individual test, much higher than other diagnostic tests in the same setting such as HIV test (3.68 US \$) or malaria microscopy (3.50 US \$) (284). Moreover, this price refers to a single blood culture bottle; as mentioned before, often two to four blood culture bottles are needed per patient, further increasing the total price. According to a panel of stakeholders, an acceptable cost for blood cultures would have to be $<10$ US $\$$, and ideally $<5$ US $\$$ (153). In the short term, donor involvement will therefore be necessary for blood cultures to be affordable.

Transport of commercial blood culture bottles from abroad is very costly and transport times are considerable, compromising shelf life. Low volumes of cultures per lab, skilled labor needs and short shelf lives further affect affordability of blood cultures in many settings (41). For this reason, many laboratories resort to in-house prepared blood culture bottles, typically containing brain-heart infusion or trypticase soy broth (41). This practice is not advised, because the origin and quality of the culture media are difficult to track down and useful additives, such as the ones described in Table 3, will not be present in the broth, compromising performance. An alternative to the commercially available bottles is to support local production of blood culture media by reference laboratories, from where they can be dispersed to smaller hospitals $(2,285)$. An exemplary initiative is the laboratory logistic and educational support

${ }^{4} \mathrm{http}$ //www.eucast.org/rapid_ast_in_blood_cultures/ 


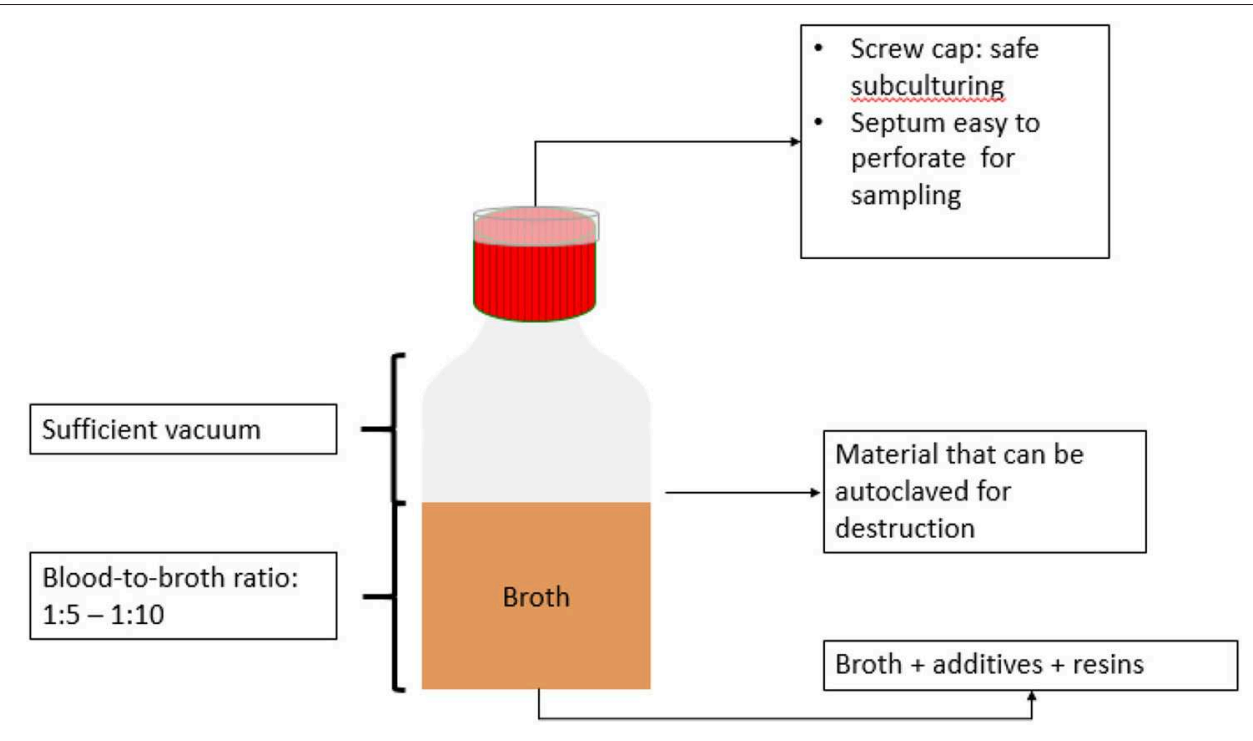

FIGURE 8 | Dissection of blood culture bottle.

provided by the Diagnostic Microbiology Development Program in Cambodia $(1)^{5}$.

\section{Need for Anaerobic Culture}

When choosing a blood culture medium to procure, it is good to remind that there is no perfect all-purpose blood culture medium. The ideal medium will depend on the expected pathogen distribution, which may vary according to the setting. Furthermore, the choice of broths will depend on whether anaerobic blood culture is deemed necessary. Most centers that analyzed the frequency of isolation of anaerobes from blood cultures have noted a declining incidence; coinciding with this, other (aerobic) organisms such as fungi, Pseudomonas species and fastidious aerobic bacteria are increasingly associated with disease (69). Some have suggested to replace routine inoculation of anaerobic media by a second aerobic bottle and reserve the anaerobic bottle only when clinically indicated or in patients at risk of anaerobic infections (286-289).

Not all hospitals, however, have seen this decrease in anaerobic bacteremia $(290,291)$. Moreover, Vena et al. found that in half of the cases of anaerobic infection, this could not have been suspected on clinical grounds (291). The choice of which bottles to use is therefore highly dependent on the specific setting, and every center is encouraged to perform its own evaluation on the need for anaerobic blood culture (69). These differences in regional incidence of anaerobic isolates from blood culture were also confirmed in a more recent review (292). In this review, it is stated that anaerobic cultures can safely be omitted in most cases, unless clinical indications for anaerobic infections are present. Moreover, identification and antibiotic susceptibility testing of anaerobic bacteria is often difficult in LMICs due to the need of specialized and generally more expensive techniques ${ }^{6}$ (293). CLSI

\footnotetext{
${ }^{5} \mathrm{http}: / / \mathrm{dmdp} . \mathrm{org}$

${ }^{6}$ http://www.eucast.org/fileadmin/src/media/PDFs/EUCAST_files/

Breakpoint_tables/v_9.0_Breakpoint_Tables.pdf
}

however still recommends that routine blood cultures include paired aerobic/anaerobic blood culture bottles, as the data are conflicting and the recommendation of limiting anaerobic blood culture bottles has never been validated by controlled clinical studies $(88,290,294,295)$. Because anaerobic bacteria are rarely recovered in pediatric patients, and usually only one bottle is sampled, the use of only aerobic bottles has been recommended in this patient population $(296,297)$.

\section{Physical Properties of the Bottle}

Equally important is the selection of the correct bottle type (Figure 8); for reasons of (bio)safety and shipping costs, plastic bottles may be preferred over glass bottles. The specific type of plastic may be important for subsequent waste management (see below). The bottle material also needs to be completely transparent to allow visual inspection for growth. Bottles have to be stored and shipped adequately to avoid scratches on the material. To increase ease of use, the septum of the bottle must be easy to perforate by a needle and the vacuum in the bottle must be sufficient to aspirate the required amount of blood $(10 \mathrm{ml}$ for adult bottles, $4 \mathrm{ml}$ for pediatric bottles). A screw cap may be more practical than other cap types in terms of waste management; after autoclavation, the fluid can then be poured out safely and the empty bottle incinerated. A screw cap is also advantageous for sampling the blood-broth mixture (e.g., for subculture), as this can avoid the use of needles.

\section{Tropicalization of Blood Culture Bottles}

Lastly, blood culture bottles should be "tropicalized", i.e., adapted to harsh environmental conditions $(1,153)$, in order to be useful for LMICs. In many LMICs, no manufacturers of invitro diagnostics are locally present, necessitating import and shipment from other countries. Therefore, shipment stability is an important factor, as is a sufficiently long shelf life. Many compounds in blood culture bottles, such as resins and SPS, may shorten shelf life, as exemplified by the much shorter shelf life 
of bottles designed for automates when compared to manual blood culture bottles. In line with requirements for malaria rapid diagnostic tests according to the Prequalification of in vitro Diagnostics (PQDx) Programme ${ }^{7}$ of the WHO, shelf life must be at least between 1.5 and 2 years. Storage temperature must be sufficiently flexible to allow storage or at least shipment at tropical temperatures (153).

\section{Signal Without Growth on Subculture}

In some cases, blood culture bottles appear "positive" (detection of bacterial growth) by a visual sign of growth such as turbidity or color change of a carbon dioxide indicator, but sub-culturing on solid media does not confirm the presence of bacteria. This phenomenon has mainly been studied for automated blood culture systems, where it can be caused by overfilling of blood culture bottles, elevated blood leukocyte counts, or antibiotic consumption by the patients before blood culture sampling (298-300). Microbiological causes are growth of Plasmodium falciparum in the blood culture bottle $(301,302)$ or growth of fastidious bacteria such as Streptococcus pneumoniae, which can exhibit rapid autolysis (i.e., self-destruction) in case of stationary growth, making it undetectable at Gram stain and subculture $(303,304)$. Other fastidious organisms, such as Campylobacter and Mycoplasma, can cause turbidity without growth, as they are difficult to stain and do not grow well on agar plates in normal conditions (266).

\section{Biosafety Concerns in Blood Cultures Need for a Biosafety Cabinet?}

A laboratory performing blood cultures usually functions as a Biosafety Level (BSL)-2 laboratory (305). When handling blood cultures, it is possible that laboratory staff comes into contact with unknown pathogens causing airborne infection. This raises the question whether laboratories in LMICs must also be equipped with a biosafety cabinet, keeping in mind the challenges in LMICs of dusty environments, lack of electricity and equipment maintenance (Table 7). A class II biosafety cabinet provides protection to laboratory staff against infectious materials as well as to biological samples against external contamination. The presence of a biosafety cabinet is generally recommended for a BSL-2 laboratory in case of likely aerosol formation or splashes (305, 306, 309). A biosafety cabinet most obviously provides protection from inhalation of infectious aerosols. For most other types of exposure, on the other hand, good personal protective equipment, such as safety goggles, face shield, gloves and masks, and the use of safe and appropriate techniques (no mouth pipetting, use of disposable loops) are sufficient. Therefore, the decision of the installation of a biosafety cabinet in a specific setting should be taken after a risk analysis, taking into account possible laboratory-acquired infections when processing blood cultures, and existing alternative methods to mitigate this risk (Table 7).

Because of gas production and possible aerosol formation, opening the blood culture bottle to perform subculture

\footnotetext{
${ }^{7}$ https://www.who.int/diagnostics_laboratory/evaluations/en/
}

TABLE 7 | Benefits, risks and limitations of a biosafety cabinet in low-resource settings.

Benefits of biosafety cabinet

Presence of a biosafety cabinet in biosafety level-2 laboratory is considered desirable according to $\mathrm{WHO}$ guidelines (305).

Procedures with a potential of generating infectious aerosols or high splash potential should be conducted within a biosafety cabinet $(305,306)$.

These may include pipetting, mixing, centrifuging, grinding, vortexing, shaking, opening of containers of infectious material with internal pressure that may be different from the ambient pressure such as blood culture bottles (306).

Pathogens of risk groups 3 and 4 are seen more frequently in low-resource settings as opposed to high-resource settings, so biosafety procedures are likely to be more important.

In case of an unexpected epidemic with a risk group 3 pathogen, having a biosafety cabinet on site can be used for outbreak investigation or other diagnostic purposes during the outbreak.

The use of pre-filters below the work bench in the biosafety cabinet or the use of door dust filters at the entrance of the laboratory can reduce the dust level within the laboratory and prolong the lifespan of the HEPA filters.

\section{Risks/limitations of biosafety cabinet}

Poor location, room air currents, decreased airflow, leaking filters, raised sashes, crowded work surfaces, and poor user technique compromise the containment capability of a biosafety cabinet (306). In those conditions, the biosafety cabinet offers a false sense of safety.

With good microbiological techniques and appropriate and consistent use of personal protective equipment (safety goggles, face shield, gloves, mask), biosafety level-2 agents can be used safely in activities conducted on the open bench, provided the potential for producing splashes or aerosols is low (306).

Workers using biosafety cabinet must be specifically trained for the use of a biosafety cabinet $(305,306)$. In low-resource settings, skilled workers are often not available,

training options are few and trained staff is difficult to retain (307).

Biosafety level-3 and 4 require additional training of the staff.

A biosafety cabinet Class II is fit for biosafety level-3 purposes but not for biosafety level-4 (305).

The biosafety cabinet must be certified when installed, whenever it is moved and then annually. In many low-resource regions, a number of practical problems prevent this from happening, most notably lack of awareness of this requirement, insufficient resources for maintenance and an absence of local competent, qualified certifiers (307, 308).

In a survey of biosafety level-2 and 3 laboratories in 7 countries in the Asia-Pacific region, $30 \%$ of biosafety cabinets tested were poorly designed, incorrectly installed, not certified, or operated improperly (308)

HEPA filters need to be replaced more frequently when working in dusty/dirty environments; replacing HEPA filters has to be done by a trained technician. In regions with low accessibility and no local manufacturers, this may be difficult to do on a regular basis.

The price of a Class II biosafety cabinet is around 10,000 euro; certifying the biosafety cabinet (which should happen at installation, annually and whenever the device is moved) costs an estimated 1,000 euro in Europe; costs in Africa or Asia are likely to be higher because of much higher transport costs, if available at all. 
substitutes a risk to the laboratory technician. Apart from measures implemented to protect the laboratory technician from this aerosol, aspects of the bottle itself can play a role in safety. For example, biphasic bottles provide opportunities of subculturing without having to open the bottle. Bottle septa that are difficult to perforate may be a risk for needle stick injuries.

\section{Waste Management}

Another important biosafety aspect is the possibility to safely destroy (decontaminate) grown blood culture bottles, for instance by autoclaving and incineration. The bacterial load of grown blood culture bottles is very high, easily reaching $10^{6}-10^{9}$ $\mathrm{CFU} / \mathrm{ml}$ (310). In case of steam autoclavation of blood culture bottles, it must be kept in mind that the fluid inside the blood culture bottle must reach $121^{\circ} \mathrm{C}$ during at least $15 \mathrm{~min}$ to ensure effective decontamination. This requires longer cycles when a higher volume of liquids (e.g., more blood culture bottles) are autoclaved, a phenomenon that is often not taken into account. Monitoring the autoclave cycle by using chemical indicators is often not sufficient, as they will only show the presence of steam but do not give an indication of the temperature inside the liquids (311). The autoclave must be validated before taken into use with the help of temperature sensors (if available) and biological indicators, preferably placed inside (non-inoculated) blood culture bottles. Given the importance of the total volume of liquids in the autoclave on the time needed to sterilize, it is important not to overfill the autoclave with liquid waste and to validate the maximum liquid load the autoclave can still safely sterilize.

Moreover, not all plastic can be safely autoclaved after use. Even if re-use of the bottles is not necessary, it is important that the bottle material can be autoclaved without posing direct biological risks by tearing and implosion of the bottles or loosening of the stopper. Polycarbonate (PC) and polypropylene (PP) bottles withstand autoclavation, however polyethylene terephthalate (PET) bottles cannot be autoclaved without substantial damage to the bottle, potentially causing spill of infectious material inside the autoclave. In case of interrupted or only partially successful cycles, this may bring the healthcare worker into contact with infectious aerosols. Therefore, other types of plastic that withstand autoclavation are preferred (like PP or PC) $)^{8}$.

\footnotetext{
$\overline{{ }^{8} \text { https://www.mynewlab.com/blog/a-guide-to-autoclaving-plastics-and-glass/ }}$
}

\section{REFERENCES}

1. Ombelet S, Ronat JB, Walsh T, Yansouni CP, Cox J, Vlieghe E, et al. Clinical bacteriology in low-resource settings: today's solutions. Lancet Infect Dis. (2018) 3099(Iso 13485):1-11. doi: 10.1016/S1473-3099(18)30093-8

2. Barbé B, Yansouni CP, Affolabi D, Jacobs J. Implementation of quality management for clinical bacteriology in low-resource settings. Clin Microbiol Infect. (2017) 23:426-33. doi: 10.1016/j.cmi.2017.05.007

3. Baron E, Dunne W, Weinstein M, Weich D, Wilson D, Yagupsky P. Cumitech 1C, Blood cultures IV. In: Baron E, editor. Cumitech 1C. Washington, DC: ASM Press (2005), 1-34.

\section{Future Research Needs}

Since the introduction of automated equipment for blood cultures, hardly any further research on manual blood culture bottles and systems has been done. This is all the more surprising given the large market share represented by manual blood culture consumables (almost double that of automated systems) and the projected substantial growth of the market for manual blood cultures in LMICs for the decades to come (27). New research and innovation should therefore also include manual blood culture methods, as we have argued elsewhere (1). The recent Unitaid Fever Diagnostic Landscape points out that short reagent shelf lives, supply chain difficulties and highly skilled labor needs drive up the cost of blood cultures in LMICs (41). As mentioned before, high cost decreases demand in these settings, resulting in low numbers of blood cultures performed. The low numbers then further contribute to higher prices per test. Moreover, the lack of critical testing volume will deny the laboratory technicians the opportunity to gain sufficient experience in processing blood cultures, thus impacting the quality of the test results and decreasing clinicians' confidence in the testing. This will in turn further drive down demand for the tests, resulting in a vicious cycle.

To break this cycle and achieve successful implementation of blood cultures in LMICs, affordable and tropicalized blood culture methods are needed. Research into these methods and close collaboration between academia, stakeholders and product developers is mandatory $(1,153)$.

\section{AUTHOR CONTRIBUTIONS}

SO did the literature review, writing of the initial draft and revisions, figure design, and project administration. $\mathrm{BB}$ contributed to literature review, revisions and provided critical review. DA, JR, PL, and OL provided critical review and commentaries. JJ and LH had the rationale for this work, contributed to literature review, and supervised manuscript revisions. LH additionally contributed to figure design. JJ additionally contributed to photography of the blood culture bottles.

\section{FUNDING}

This work was supported by the InBev-Baillet Latour (IBL) Fund for the Bacterial Infections in the Tropics (BIT) research cluster at the Institute of Tropical Medicine (ITM), Antwerp, Belgium.

4. Yagupsky P, Nolte FS. Quantitative aspects of septicemia. Clin Microbiol Rev. (1990) 3:269-79. doi: 10.1128/CMR.3. 3.269

5. Vlieghe ER, Phe T, De Smet B, Chhun Veng H, Kham C, Lim $\mathrm{K}$, et al. Bloodstream Infection among adults in Phnom Penh, Cambodia: key pathogens and resistance patterns. PLoS ONE. (2013) 8:1-9. doi: 10.1371/journal.pone.0059775

6. Cockerill FR, Wilson JW, Vetter EA, Goodman KM, Torgerson CA, Harmsen WS, et al. Optimal testing parameters for blood cultures. Clin Infect Dis. (2004) 38:1724-30. doi: $10.1086 / 4$ 21087 
7. Raza SS, Sultan OW, Sohail MR. Gram-negative bacterial endocarditis in adults: State-of-the-heart. Expert Rev Anti Infect Ther. (2010) 8:87985. doi: $10.1586 /$ eri. 10.76

8. Eliakim-Raz N, Bates DW, Leibovici L. Predicting bacteraemia in validated models-a systematic review. Clin Microbiol Infect. (2015) 21:295301. doi: 10.1016/j.cmi.2015.01.023

9. Paul M, Andreassen S, Nielsen AD, Tacconelli E, Almanasreh N, Fraser A, et al. Prediction of bacteremia using TREAT, a computerized decisionsupport system. Clin Infect Dis. (2006) 42:1274-82. doi: 10.1086/5 03034

10. Lee J, Hwang SS, Kim K, Jo YH, Lee JH, Kim J, et al. Bacteremia prediction model using a common clinical test in patients with community-acquired pneumonia. Am J Emerg Med. (2014) 32:700-4. doi: 10.1016/j.ajem.2014.04.010

11. Jones GR, Lowes J. The systemic inflammatory response syndrome as a predictor of bacteraemia and outcome from sepsis. QJM. (1996) 89:51522. doi: 10.1093/qjmed/89.7.515

12. Shapiro NI, Wolfe RE, Wright SB, Moore R, Bates D. Who needs a blood culture? A prospectively derived and validated prediction rule. J Emerg Med. (2008) 35:255-64. doi: 10.1016/j.jemermed.2008.04.001

13. Seymour CW, Liu VX, Iwashyna TJ, Brunkhorst FM, Rea TD, Scherag A, et al. Assessment of clinical criteria for sepsis for the third international consensus definitions for sepsis and septic shock (sepsis-3). JAMA. (2016) 315:762-74. doi: 10.1001/jama.2016.0288

14. Coburn B, Morris AM, Tomlinson G, Detsky AS. Does this adult patient with suspected bacteremia require blood cultures. JAMA. (2012) 308:50211. doi: 10.1001/jama.2012.8262

15. Brown JD, Chapman S, Ferguson PE. Blood cultures and bacteraemia in an Australian emergency department: evaluating a predictive rule to guide collection and their clinical impact. EMA. (2017) 29:5662. doi: 10.1111/1742-6723.12696

16. Hodgson LE, Dragolea N, Venn R, Dimitrov BD, Forni LG. An external validation study of a clinical prediction rule for medical patients with suspected bacteraemia. Emerg Med J. (2016) 33:1249. doi: 10.1136/emermed-2015-204926

17. Singer M, Deutschman CS, Seymour CW, Shankar-Hari M, Annane D, Bauer $\mathrm{M}$, et al. The third international consensus definitions for sepsis and septic shock. JAMA. (2016) 315:801-10. doi: 10.1001/jama.2016.0287

18. Falay D, Kuijpers LMF, Phoba MF, De Boeck H, Lunguya O, Vakaniaki E, et al. Microbiological, clinical and molecular findings of non-typhoidal Salmonella bloodstream infections associated with malaria, Oriental Province, Democratic Republic of the Congo. BMC Infect Dis. (2016) 16:114. doi: 10.1186/s12879-016-1604-1

19. Kalonji LM, Post A, Phoba MF, Falay D, Ngbonda D, Muyembe JJ, et al. Invasive salmonella infections at multiple surveillance sites in the Democratic Republic of the Congo, 2011-2014. Clin Infect Dis. (2015) 61(Suppl 4):S346-53. doi: 10.1093/cid/civ713

20. World Health Organization. Recommendations for Management of Common Childhood Conditions. (2012). 84 p. Available online at: https://www. who.int/maternal_child_adolescent/documents/management_childhood_ conditions/en/

21. Semov A, Marcotte R, Semova N, Ye X, Wang E. Microarray analysis of E-box binding-related gene expression in young and replicatively senescent human fibroblasts. Anal Biochem. (2002) 302:38-51. doi: 10.1006/abio.2001.5515

22. Martin GR, MacLennan SJ. Analysis of the 5-HT receptor in rabbit saphenous vein examplifies the problems of using exclusion criteria for receptor classification. Naunyn Schmiedebergs Arch Pharmacol. (1990) 342:111-9. doi: 10.1007/BF00166952

23. Lamy B, Dargière $S$, Arendrup MC, Parienti JJ, Tattevin P. How to optimize the use of blood cultures for the diagnosis of bloodstream infections? A stateof-the art. Front Microbiol. (2016) 7:1-13. doi: 10.3389/fmicb.2016.00697

24. El-din AAK, Mohamed MA, Gad WH. Prevalence of microbial pathogens in blood cultures: an etiological and histopathological study. J Taibah Univ Sci. (2010) 3:23-32. doi: 10.1016/S1658-3655(12)60017-X

25. Elantamilan D, Lyngdoh VW, Khyriem A, Rajbongshi J, Bora I, Devi ST, et al. Comparative evaluation of the role of single and multiple blood specimens in the outcome of blood cultures using BacT/ALERT 3D (automated) blood culture system in a tertiary care hospital. Indian J Crit Care Med. (2016) 20:530-3. doi: 10.5005/ijccm-20-9-530

26. Ahmad A, Iram S, Hussain S, Yusuf NW. Diagnosis of paediatric sepsis by automated blood culture system and conventional blood culture. J Pak Med Assoc. (2017) 67:192-5.

27. Grand View Research. Blood Culture Tests: Market Analysis \& Segment Forecast From 2014 - 2025. Grand View Research, Inc. (2018).

28. Peeling RW, Holmes KK, Mabey D, Ronald A. Rapid tests for sexually transmitted infections (STIs): the way forward. Sex Transm Infect. (2006) 82(Suppl. 5):v1-6. doi: 10.1136/sti.2005.017251

29. Land KJ, Boeras DI, Chen X-S, Ramsay AR, Peeling RW. REASSURED diagnostics to inform disease control strategies, strengthen health systems and improve patient outcomes. Nat Microbiol. (2019) 4:46-54. doi: 10.1038/s41564-018-0295-3

30. Fleischmann-Struzek C, Goldfarb DM, Schlattmann P, Schlapbach LJ, Reinhart K, Kissoon N. The global burden of paediatric and neonatal sepsis: a systematic review. Lancet Respir Med. (2018) 6:168-70. doi: 10.1016/S2213-2600(18)30063-8

31. Vincent JL, Marshall JC, Namendys-Silva SA, François B, Martin-Loeches I, Lipman J, et al. Assessment of the worldwide burden of critical illness: the Intensive Care Over Nations (ICON) audit. Lancet Respir Med. (2014) 2:380-6. doi: 10.1016/S2213-2600(14)70061-X

32. Fleischmann C, Scherag A, Adhikari NKJ, Hartog CS, Tsaganos T, Schlattmann P, et al. Assessment of global incidence and mortality of hospital-treated sepsis current estimates and limitations. Am J Respir Crit Care Med. (2016) 193:259-72. doi: 10.1164/rccm.201504-0781OC

33. Otu A, Elston J, Nsutebu E. Sepsis in Africa: Practical steps to stem the tide. Pan Afr Med J. (2015) 21:1-4. doi: 10.11604/pamj.2015.21.323 .6462

34. Petti CA, Polage CR, Quinn TC, Ronald AR, Sande MA. Laboratory medicine in Africa: a barrier to effective health care. Clin Infect Dis. (2006) 42:377-82. doi: 10.1086/499363

35. Rhee C, Dantes R, Epstein L, Murphy DJ, Seymour CW, Iwashyna TJ, et al. Incidence and trends of sepsis in US hospitals using clinical vs claims data, 2009-2014. JAMA. (2017) 318:1241-9. doi: 10.1001/jama.2017.13836

36. Stevenson EK, Rubenstein AR, Radin GT, Wiener RS, Walkey A. Two decades of mortality trends among patients with severe sepsis: a comparative meta-analysis. Crit Care Med. (2015) 42:625-31. doi: 10.1097/CCM.0000000000000026

37. Dellinger RP, Levy MM, Rhodes A, Annane D, Gerlach H, Opal SM, et al. Surviving Sepsis Campaign: international guidelines for management of severy sepsis and septic shock: 2012. Crit Care Med. (2013) 41:580637. doi: $10.1007 / \mathrm{s} 00134-012-2769-8$

38. Kumar A, Roberts D, Wood KE, Light B, Parrillo JE, Sharma S, et al. Duration of hypotension before initiation of effective antimicrobial therapy is the critical determinant of survival in human septic shock. Crit Care Med. (2006) 34:1589-96. doi: 10.1097/01.CCM.0000217961.75225.E9

39. Evans JA, Adusei A, Timmann C, May J, Mack D, Agbenyega T, et al. High mortality of infant bacteraemia clinically indistinguishable from severe malaria. QJM. (2004) 97:591-7. doi: 10.1093/qjmed/hch093

40. Reyburn H, Mbatia R, Drakeley C, Carneiro I, Mwakasungula E, Mwerinde $\mathrm{O}$, et al. Overdiagnosis of malaria in patients with severe febrile illness in Tanzania: a prospective study. Br Med J. (2004) 329:12125. doi: 10.1136/bmj.38251.658229.55

41. Jennifer DU. Technology Landscape: Fever Diagnostic Technology Landscape. Geneva: Unitaid (2018).

42. Guiraud I, Post A, Diallo SN, Lompo P, Maltha J, Thriemer K, et al. Population-based incidence, seasonality and serotype distribution of invasive salmonellosis among children in Nanoro, rural Burkina Faso. PLoS ONE. (2017) 12:1-17. doi: 10.1371/journal.pone.0178577

43. Church J, Maitland K. Invasive bacterial co-infection in African children with Plasmodium falciparum malaria: a systematic review. BMC Med. (2014) 12:1-16. doi: 10.1186/1741-7015-12-31

44. Musicha P, Cornick JE, Bar-Zeev N, French N, Masesa C, Denis $\mathrm{B}$, et al. Trends in antimicrobial resistance in bloodstream infection isolates at a large urban hospital in Malawi (1998-2016): a surveillance study. Lancet Infect Dis. (2017) 17:1042-52. doi: 10.1016/S1473-3099(17) 30394-8 
45. Reddy EA, Shaw AV, Crump JA. Community-acquired bloodstream infections in Africa: a systematic review and meta-analysis. Lancet Infect Dis. (2010) 10:417-32. doi: 10.1016/S1473-3099(10)70072-4

46. Deen J, von Seidlein L, Andersen F, Elle N, White NJ, Lubell Y. Communityacquired bacterial bloodstream infections in developing countries in south and southeast Asia: a systematic review. Lancet Infect Dis. (2012) 12:4807. doi: 10.1016/S1473-3099(12)70028-2

47. Crump JA, Sjölund-Karlsson M, Gordon MA, Parry CM. Epidemiology, clinical presentation, laboratory diagnosis, antimicrobial resistance, and antimicrobial management of invasive Salmonella infections. Clin Microbiol Rev. (2015) 28:901-37. doi: 10.1128/CMR.00002-15

48. Cooper GS, Havlir DS, Shlaes DM, Salata RA. Polymicrobial bacteremia in the late 1980s: predictors of outcome and review of the literature. Medicine (Baltimore). (1990) 69:114-22. doi: 10.1097/00005792-19906902 0-00005

49. Sutter D, Stagliano D, Braun LR, Williams F, Arnold J, Ottolini M, et al. Polymicrobial bloodstream infection in pediatric patients: risk factors, microbiology, and antimicrobial management. Pediatr Infect Dis J. (2008) 27:400-5. doi: 10.1097/INF.0b013e31816591be

50. Weinstein MP, Reller BL, Murphy JR. Clinical importance of polymicrobial bacteremia. Diagn Microbiol Infect Dis. (1986) 5:185-96. doi: 10.1016/0732-8893(86)90001-5

51. Roselle GA, Watanakunakorn C. Polymicrobial bacteremia. JAMA. (1979) 242:2411-3. doi: 10.1001/jama.242.22.2411

52. Lin JN, Lai CH, Chen YH, Chang LL, Lu PL, Tsai SS, et al. Characteristics and outcomes of polymicrobial bloodstream infections in the emergency department: a matched case-control study. Acad Emerg Med. (2010) 17:1072-9. doi: 10.1111/j.1553-2712.2010.00871.x

53. Wisplinghoff H, Bischoff T, Tallent SM, Seifert H, Wenzel RP, Edmond MB. Nosocomial bloodstream infections in US hospitals: analysis of 24,179 cases from a prospective nationwide surveillance study. Clin Infect Dis. (2004) 39:309-17. doi: 10.1086/421946

54. Pavlaki M, Poulakou G, Drimousis P, Adamis G, Apostolidou E, Gatselis NK, et al. Polymicrobial bloodstream infections: epidemiology and impact on mortality. J Glob Antimicrob Resist. (2013) 1:20712. doi: 10.1016/j.jgar.2013.06.005

55. WHO. Global Antimicrobial Resistance Surveillance System. Manual for Early Implementation. WHO (2015). p. 1-44. Available online at: http://www. who.int/drugresistance/en/\%5Cnwww.who.int/about/licensing/copyright_ form/en/index.\%5Cnhttp://apps.who.int/iris/bitstream/10665/188783/1/ 9789241549400_eng.pdf?ua=1

56. Barenfanger J, Graham DR, Kolluri L, Sangwan G, Lawhorn J, Drake CA, et al. Decreased mortality associated with prompt gram staining of blood cultures. Am J Clin Pathol. (2008) 130:870-6. doi: 10.1309/AJCPVMDQU2ZJDPBL

57. Munson EL, Diekema DJ, Beekmann SE, Chapin KC, Doern GV. Detection and treatment of bloodstream infection: laboratory reporting and antimicrobial management. J Clin Microbiol. (2003) 41:495-7. doi: 10.1128/JCM.41.1.495-497.2003

58. Cunney RJ, McNamara EB, Alansari N, Loo B, Smyth EG. The impact of blood culture reporting and clinical liaison on the empiric treatment of bacteraemia. J Clin Pathol. (1997) 50:1010-2. doi: 10.1136/jcp.50. 12.1010

59. Uehara Y, Yagoshi M, Tanimichi Y, Yamada H, Shimoguchi K, Yamamoto $\mathrm{S}$, et al. Impact of reporting gram stain results from blood culture bottles on the selection of antimicrobial agents. Am J Clin Pathol. (2009) 132:1825. doi: 10.1309/AJCP0H2DAMBXZUSS

60. Beekmann SE, Diekema DJ, Chapin KC, Doern GV. Effects of rapid detection of bloodstream infections on length of hospitalization and hospital charges effects of rapid detection of bloodstream infections on length of hospitalization and hospital charges. J Clin Microbiol. (2003) 41:311925. doi: 10.1128/JCM.41.7.3119-3125.2003

61. Schønheyder HHT. The impact of the first notification of positive blood cultures on antibiotic therapy. A one-year survey. APMIS. (1995) 103:37-44. doi: 10.1111/j.1699-0463.1995.tb0 1077.x

62. Fidelman P, Rygalski K, Riley J, Hernandez D, Wolk D. Characterization of clinical improvements after implementation of new blood culture strategy for patients with severe sepsis. Open Forum Infect Dis. (2016) 3:1875. doi: 10.1093/ofid/ofw172.1423

63. Benbachir M, Editor C, Outline T. Role of the microbiology laboratory in infection control. In: Bearman GML, editor. Guide to Infection Control in the Hospital. Brookline, MA: International Society for Infectious Diseases (2018).

64. De Smet B, Veng C, Kruy L, Kham C, van Griensven J, Peeters C, et al. Outbreak of Burkholderia cepacia bloodstream infections traced to the use of Ringer lactate solution as multiple-dose vial for catheter flushing, Phnom Penh, Cambodia. Clin Microbiol Infect. (2013) 19:8327. doi: 10.1111/1469-0691.12047

65. World Health Organization (WHO). Report on the Burden of Endemic Health Care-Associated Infection Worldwide. WHO Library Cataloguing Data (2011).

66. Friedman ND, Kaye KS, Stout JE, Mcgarry SA, Trivette SL, Briggs JP, et al. Health care - associated bloodstream infections in adults: a reason to change the accepted definition of community - acquired infections. Ann Fam Med. (2002) 791-8. doi: 10.7326/0003-4819-137-10-200211190-00007

67. Lenz R, Leal JR, Church DL, Gregson DB, Ross T, Laupland KB. The distinct category of healthcare associated bloodstream infections. BMC Infect Dis. (2012) 12:85. doi: 10.1186/1471-2334-12-85

68. Rodríguez-Baño J, López-Prieto MD, Portillo MM, Retamar P, Natera C, Nuño E, et al. Epidemiology and clinical features of community-acquired, healthcare-associated and nosocomial bloodstream infections in tertiarycare and community hospitals. Clin Microbiol Infect. (2010) 16:140813. doi: 10.1111/j.1469-0691.2010.03089.x

69. Reimer LG, Wilson ML, Weinstein MP. Update on detection of bacteremia and fungemia. Clin Microbiol Rev. (1997) 10:444-65. doi: 10.1128/CMR.10.3.444

70. Leber AL. Clinical Microbiology Procedures Handbook. Washington, DC: ASM Press (2016).

71. Murray PR. Determination of the optimum incubation period of blood culture broths for the detection of clinically significant septicemia. J Clin Microbiol. (1985) 21:481-5.

72. Weinstein MP. Emerging data indicating that extended incubation of blood cultures has little clinical value. Clin Infect Dis. (2005) 41:16812. doi: $10.1086 / 497603$

73. Isenbergh. Clinical Microbiology Procedures Handbook. ASM Press (2004).

74. Miller JM, Binnicker MJ, Campbell S, Carroll KC, Chapin KC, Gilligan PH, et al. IDSA guideline a guide to utilization of the microbiology laboratory for diagnosis of infectious diseases: 2018 update by the Infectious Diseases Society of America and the American Society for microbiology. Clin Infect Dis. (2018) 67:1-94. doi: 10.1093/cid/ciy584

75. Bourbeau PP, Pohlman JK. Three days of incubation may be sufficient for routine blood cultures with BacT/Alert FAN blood culture bottles three days of incubation may be sufficient for routine blood cultures with BacT/Alert FAN blood culture bottles. J Clin Microbiol. (2001) 39:207982. doi: 10.1128/JCM.39.6.2079-2082.2001

76. Shanson C. An experimental assessment of different anaerobic blood culture methods. J Clin Pathol. (1974) 27:273-9. doi: 10.1136/jcp.27.4.273

77. Hansen GT. Laboratory blood cultures: past, present, and future. Clin Microbiol Newsl. (2016) 38:119-28. doi: 10.1016/j.clinmicnews.2016.07.001

78. Washington 2nd JA. Evaluation of two commercially available media for detection of bacteremia. Appl Microbiol. (1971) 2:604-7.

79. Wilson ML. General principle in th laboratory detection of bacteremia and fungemia. Clin Lab Med. (1994) 14:6982. doi: 10.1016/S0272-2712(18)30395-0

80. Reimer L, Reller B, Wang W, Mirrett S. Evaluation of modified trypticase soy broth versus supplemented peptone broth in the detection of bacteremia and fungemia. Eur J Clin Microbiol Infect Dis. (1988) 7:3847. doi: 10.1007/BF01962342

81. Gross PA, Fryda R, Reilly K. Comparative evaluation of different types of blood culture media for isolation of aerobes. J Clin Microbiol. (1977) 6:362-6.

82. Reimer LG, Reller LB. Effect of sodium polyanetholesulfonate and gelatin on the recovery of Gardnerella vaginalis from blood culture media. J Clin Microbiol. (1985) 21:686-8.

83. Reimer L, Reller L, Wang W, Mirrett S. Controlled evaluation of trypticase soy broth with and without gelatin and yeast extract in the detection 
of bacteremia and fungemia. Diagn Microbiol Infect Dis. (1987) 8:1924. doi: 10.1016/0732-8893(87)90042-3

84. Weinstein MP, Reller LB, Mirrett S, Reimer LG, Wang WL, Stratton CW. Controlled evaluation of modified radiometric blood culture medium supplemented with gelatin for detection of bacteremia and fungemia. J Clin Microbiol. (1987) 25:1373-5.

85. Stratton CW, Weinstein MP, Mirrett S, Paisley J, Lauer BA, Reller LB. Controlled evaluation of blood culture medium containing gelatin and V-factor-analog for detection of septicemia in children. J Clin Microbiol. (1988) 26:747-9.

86. McDonald J, Knowles K, Sorger S. Assessment of gelatin supplementation of PEDS Plus BACTEC blood culture medium. Diagn Microbiol Infect Dis. (1993) 17:193-6. doi: 10.1016/0732-8893(93)90095-O

87. Artman M, Domenech E, Weiner M. Growth of Haemophilus influenzae in simulated blood cultures supplemented with Hemin and NAD. J Clin M. (1983) 18:376-9.

88. Wilson M, Mitchell M, Morris A, Murray P, Reimer L, Barth RL, et al. Principles and Procedures for Blood Cultures: Approved Guideline, Vol. 27. Clinical and Laboratory Standards Institute (2007). 60 p. Available online at: http://shop.clsi.org/c.1253739/site/Sample_pdf/M47A_sample.pdf \%5Cnhttp://www2.edah.org.tw/cp/study/04_1020308CLSIM47.pdf

89. Ganguli LA, Turton LJ, Tillotson GS. Evaluation of fastidious anaerobe broth as a blood culture medium. I Clin Pathol. (1982) 35:45861. doi: $10.1136 /$ jcp. 35.4 .458

90. Doern GV. Manual blood culture systems and the antimicrobial removal device. Clin Lab Med. (1994) 14:13347. doi: 10.1016/S0272-2712(18)30400-1

91. Shanson DC, Barnicoat M. An experimental comparison of Thiol broth with Brewer's thioglycollate for anaerobic blood cultures. J Clin Pathol. (1975) 28:407-9. doi: 10.1136/jcp.28.5.407

92. Auckenthaler R, Ilstrup DM, Washington JA. Comparison of recovery of organisms from blood cultures diluted 10\% (volume/volume) and $20 \%$ (volume/volume). J Clin Microbiol. (1982) 15:860-4.

93. Kennaugh JK, Gergory WW, Powell KR, Hendley JO. The effect of dilution during culture on detection of low concentrations of bacteria in blood. Pediatr Infect Dis. (1984) 3:317-8. doi: 10.1097/00006454-19840700000008

94. Reller LB. Recent and innovative methods for detection of bacteremia and fungemia. Am J Med. (1983) 26-30. doi: 10.1016/0002-9343(83)90069-4

95. Elliott TS, Stevens CM, Macrae F, Hart IT, Healing DE, Palmer M, et al. Improved recovery of antibiotic-stressed microorganisms on inclusion of saponin in aerobic blood culture media. Eur J Clin Microbiol Infect Dis. (1998) 17:566-9. doi: 10.1007/BF01708620

96. Rohner P, Pepey B, Auckenthaler R. Advantage of combining resin with lytic BACTEC blood culture media. J Clin Microbiol. (1997) 35:2634-8.

97. Scheer C, Fuchs C, Gründling M, Volmer M, Bast J, Bohnert J, et al. Impact of antibiotic administration on blood culture positivity at the beginning of sepsis: a prospective clinical cohort study. Clin Microbiol Infect. (2019) 25:326-31. doi: 10.1016/j.cmi.2018.05.016

98. Hummel M, Warga C, Hof H, Hehlmann R, Buchheidt D. Diagnostic yield of blood cultures from antibiotic-naïve and antibiotically treated patients with haematological malignancies and high-risk neutropenia. Scand J Infect Dis. (2009) 41:650-5. doi: 10.1080/00365540903062150

99. Rodriguez F, Lorian V. Antibacterial activity in blood cultures. J Clin Microbiol. (1985) 21:262-3.

100. Pazin G, Saul S, Thompson M. Blood culture positivity: suppression by outpatient antibiotic therapy in patients with bacterial endocarditis. Arch Intern Med. (1982) 142:263-8. doi: 10.1001/archinte.1982.00340150063013

101. Maltha J, Guiraud I, Kaboré B, Lompo P, Ley B, Bottieau E, et al. Frequency of severe malaria and invasive bacterial infections among children admitted to a rural hospital in Burkina Faso. PLoS ONE. (2014) 9:18. doi: 10.1371/journal.pone.0089103

102. Radyowijati A, Haak H. Improving antibiotic use in low-income countries: an overview of evidence on determinants. Soc Sci Med. (2003) 57:73344. doi: 10.1016/S0277-9536(02)00422-7

103. Morgan DJ, Okeke IN, Laxminarayan R, Perencevich EN. Non-prescription antimicrobial use worldwide: a systematic review. Lancet Infect Dis. (2013) 11:692-701. doi: 10.1016/S1473-3099(11)70054-8
104. Pearson M, Doble A, Glogowski R, Ibezim S, Lazenby T, Haile-Redai A, et al. Antibiotic Prescribing and Resistance: Views From Low-and Middle Income Prescribing and Dispensing Professionals. Report to the World Health Organization, researched and compiled by students and staff of the Antimicrobial Resistance Centre at the London School of Hygiene and Tropical Medicine (LSHTM) (2017).

105. Appleman MD, Swinney RS, Heseltine PNR. Evaluation of the antibiotic removal device. J Clin Microbiol. (1982) 15:278-81.

106. Washington JI. Blood cultures: principles and techniques. Mayo Clin Proc. (1975) 50:91-8.

107. Washington JA. Collection, transport and processing of blood cultures. Clin Lab Med. (1994) 14:59-68. doi: 10.1016/S0272-2712(18)30394-9

108. Wilson ML, Weinstein MP, Mirrett S, Reimer LG, Feldman RJ, Chuard $\mathrm{CR}$, et al. Controlled evaluation of BacT/alert standard anaerobic and FAN anaerobic blood culture bottles for the detection of bacteremia and fungemia. J Clin Microbiol. (1995) 33:2265-70.

109. Weinstein MP, Mirrett S, Reimer LG, Wilson ML, Smith-elekes S, Chuard $\mathrm{CR}$, et al. Controlled evaluation of BacT/alert standard aerobic and FAN Aerobic blood culture bottles for detection of bacteremia and fungemia. $J$ Clin Microbiol. (1995) 33:978-81.

110. Mitteregger D, Barousch W, Nehr M, Kundi M, Zeitlinger M, Makristathis A, et al. Neutralization of antimicrobial substances in new BacT/alert FA and FN plus blood culture bottles. J Clin Microbiol. (2013) 51:153440. doi: 10.1128/JCM.00103-13

111. Lovern D, Katzin B, Johnson K, Broadwell D, Miller E, Gates A, et al. Antimicrobial binding and growth kinetics in BacT/ALERT $®$ FA plus and BACTEC $®$ Aerobic/F Plus blood culture media. Eur J Clin Microbiol Infect Dis. (2016) 35:2033-6. doi: 10.1007/s10096-016-2759-9

112. Spaargaren J, Van Boven CPA, Voorn GP. Effectiveness of resins in neutralizing antibiotic activities in Bactec Plus Aerobic/F culture medium effectiveness of resins in neutralizing antibiotic activities in Bactec Plus Aerobic/F culture medium. J Clin Microbiol. (1998) 36:3731-3.

113. Rozdzinski E, Kirchner S, Kern W. Removal of the antibacterial activity of TrimethoprimSulfamethoxazole, Ofloxacin and Zidovudine by BACTEC resin-containing blood culture medium. Zentralblatt Bakteriol. (1991) 275:320-6. doi: 10.1016/S0934-8840(11)80295-1

114. Doern CD, Mirrett S, Halstead D, Abid J, Okada P, Reller LB. Controlled clinical comparison of new pediatric medium with adsorbent polymeric beads (PF Plus) versus charcoal-containing PF medium in the bact/alert blood culture system. J Clin Microbiol. (2014) 52:1898900. doi: 10.1128/JCM.00175-14

115. Miller NS, Rogan D, Orr BL, Whitney D. Comparison of BD bactec plus blood culture media to versaTREK redox blood culture media for detection of bacterial pathogens in simulated adult blood cultures containing therapeutic concentrations of antibiotics. J Clin Microbiol. (2011) 49:16247. doi: 10.1128/JCM.01958-10

116. Flayhart D, Borek AP, Wakefield T, Dick J, Carroll KC. Comparison of Bactec Plus blood culture media to BacT/Alert FA blood culture media for detection of bacterial pathogens in samples containing therapeutic levels of antibiotics. J Clin Microbiol. (2007) 45:816-21. doi: 10.1128/JCM.02064-06

117. Sullivan KV, Turner NN, Lancaster DP, Shah AR, Chandler LJ, Friedman DF, et al. Superior sensitivity and decreased time to detection with the bactec peds Plus/F system compared to the BacT/Alert pediatric FAN blood culture system. J Clin Microbiol. (2013) 51:4083-6. doi: 10.1128/JCM.02205-13

118. Viganò EF, Vasconi E, Agrappi C, Clerici P, Melloni P. Use of simulated blood cultures for antibiotic effect on time to detection of the two blood culture systems BacT/ALERT and BACTECTM 9240. New Microbiol. (2004) 27:235-48.

119. Ruiz-Giardín JM, Martin-Díaz RM, Jaqueti-Aroca J, Garcia-Arata I, San Martín-López JV, Sáiz-Sánchez Buitrago M. Diagnosis of bacteraemia and growth times. Int J Infect Dis. (2015) 41:6-10. doi: 10.1016/j.ijid.2015.10.008

120. Clancy CJ, Nguyen MH. Finding the "Missing $50 \%$ " of invasive candidiasis: how nonculture diagnostics will improve understanding of disease spectrum and transform patient care. Clin Infect Dis. (2013) 56:128492. doi: $10.1093 / \mathrm{cid} / \mathrm{cit} 006$

121. Datcu R, Boel J, Jensen I, Arpi M. Comparison of BACTEC ${ }^{\mathrm{TM}}$ blood culture media for the detection of fungemia. Eur J Clin Microbiol Infect Dis. (2017) 36:131-7. doi: 10.1007/s10096-016-2781-y 
122. Ericson E, Klingspor L, Ullberg M, Ozenci V. Clinical comparison of the Bactec Mycosis IC/F, BacT/Alert FA, and BacT/Alert FN blood culturevials for the detection of candidemia. Diagn Microbiol Infect Dis. (2012) 73:1536. doi: 10.1016/j.diagmicrobio.2012.02.020

123. Nylén T, Saeedi B, Borg C, Ullberg M, Ozenci V. The performance of 4 different supplements and 5 blood culture bottles types in detection of bacteria and Candida spp. in simulated sterile body fluid cultures. Diagn Microbiol Infect Dis. (2013) 77:1-4. doi: 10.1016/j.diagmicrobio.2013.05.017

124. Azar MM, Hage CA. Laboratory diagnostics for histoplasmosis. J Clin Microbiol. (2017) 55:1612-20. doi: 10.1128/JCM.02430-16

125. Parikh HR, De AS, Baveja SM. Comparison of the Lysis Centrifugation method with the conventional blood culture method in cases of sepsis in a tertiary care hospital. J Lab Physicians. (2012) 4:8993. doi: 10.4103/0974-2727.105588

126. Henry NK, McLimans CA, Wright AH, Thompson RL, Wilson WR, Washington JA. Microbiological and clinical evaluation of the isolator LysisCentrifugation Blood Culture Tube. J Clin Microbiol. (1983) 17:864-9.

127. Thomson RB, Vanzo SJ, Henry NK, Guenther KL, Washington JA. Contamination of cultures processed with the Isolator lysis-centrifugation blood culture tube. J Clin Microbiol. (1984) 19:97-9.

128. Agy MB, Wallis CK, Plorde JJ, Carlson LC, Coyle MB. Evaluation of four mycobacterial blood culture media: BACTEC 13A, isolator/BACTEC 12B, isolator/middlebrook agar, and a biphasic medium. Diagn. (1989) 12:3038. doi: 10.1016/0732-8893(89)90094-1

129. Archibald LK, McDonald LC, Addison RM, McKnight C, Byrne T, Dobbie $\mathrm{H}$, et al. Comparison of BACTEC MYCO/F LYTIC and WAMPOLE ISOLATOR 10 (Lysis-centrifugation) systems for detection of bacteremia, mycobacteremia, and fungemia in a developing country. J Clin Microbiol. (2000) 38:2994-7.

130. Castaneda MR. A practical method for routine blood cultures in brucellosis. Proc Soc Exp Biol Med. (1947) 64:114. doi: 10.3181/00379727-64-15717

131. Weckbach LS, Staneck JL. Performance characteristics of a commercially prepared biphasic blood culture bottle. J Clin Microbiol. (1986) 23:700-3.

132. Malmvall BE, Alestig K, Brorson JE, Elgefors B. Clinical evaluation of lysis-centrifugation technique and a biphasic bottle system for blood culture. Scand J Infect Dis. (1985) 17:401-6. doi: 10.3109/1381345850905 8781

133. Kelly MT, Buck GE, Fojtasek MF. Evaluation of a lysis-centrifugation and biphasic bottle blood culture system during routine use. J Clin Microbiol. (1983) 18:554-7.

134. Hall MM, Mueske CA, Ilstrup DM, Washington JA. Evaluation of a biphasic medium for blood cultures. J Clin Microbiol. (1979) 10:673-6.

135. Brook I, Gruenwald L. Detection of bacteraemia in children seen in the outpatient department: a comparison of conventional blood culture methods and a Castaneda blood culture. Microbios. (1982) 35:17-20.

136. Degaute C, Chapusette P, Vanoudenhove J, Pierret C, Serruys-Schoutens E. Comparison of a biphasic medium plus routine early subculture with a slide blood culture system. Eur J Clin Microbiol Infect Dis. (1985) 4:47882. doi: 10.1007/BF02014428

137. Ispahani P, Pearson NJ, Donald F. Blood cultures: eight years' experience of a conventional in-house system and trends in antimicrobial susceptibilities. Med Lab Sci. (1989) 46:295-304.

138. Henry NK, Grewell CM, McLimans CA, Washington JA. Comparison of the Roche septi-chek blood culture bottle with a brain heart infusion biphasic medium bottle and with a tryptic soy broth bottle. J Clin Microbiol. (1984) 19:315-7.

139. Pfaller MA, Sibley TK, Westfall LM, Hoppe-Bauer JE, Keating MA, Murray PR. Clinical laboratory comparison of a slide blood culture system with a conventional broth system. J Clin Microbiol. (1982) 16:525-30.

140. Weinstein MP, Reller LB, Mirrett S, Wang WL, Alcid DV. Clinical comparison of an agar slide blood culture bottle with tryptic soy broth and a conventional blood culture bottle with supplemented peptone broth. J Clin Microbiol. (1985) 21:815-8.

141. Bryan LE. Comparison of a slide blood culture system with a supplemented peptone broth culture method. J Clin Microbiol. (1981) 14:389-92.

142. Arpi M, Bentzon MW, Jensen J, Frederiksen W. Importance of blood volume cultured in the detection of bacteremia. Eur J Microbiol Infect Dis. (1989) 8:838-42. doi: 10.1007/BF02185857
143. Ilstrup DM, Washington JA. The importance of volume of blood cultured in the detection of bacteremia and fungemia. Diagn Microbiol Infect Dis. (1983) 1:107-10. doi: 10.1016/0732-8893(83)90039-1

144. Tenney JH, Reller LB, Mirrett S, Wang WL, Weinstein MP. Controlled evaluation of the volume of blood cultured in detection of bacteremia and fungemia. J Clin Microbiol. (1982) 15:558-61.

145. Patel R, Vetter EA, Harmsen WS, Schleck CD, Fadel HJ, Cockerill FR. Optimized pathogen detection with 30- compared to 20-milliliter blood culture draws. J Clin Microbiol. (2011) 49:4047-51. doi: 10.1128/JCM.01314-11

146. Mermel LA, Maki DG. Detection of bacteremia in adults: consequences of culturing an inadequate volume of blood. Ann Intern Med. (1993) 119:2702. doi: 10.7326/0003-4819-119-4-199308150-00003

147. Lee A, Mirrett S, Reller LB, Weinstein MP. Detection of bloodstream infections in adults: How many blood cultures are needed? J Clin Microbiol. (2007) 45:3546-8. doi: 10.1128/JCM.01555-07

148. Shoji K, Tsuboi N, Arakawa R, Ide K. Continuous monitoring and feedback optimizes blood volume inoculated into culture bottles in the pediatric intensive care unit. J Pediatr Infect Dis Soc. (2018) 1:4-7. doi: 10.1093/jpids/piy061

149. Willems E, Smismans A, Cartuyvels R, Coppens G, Van Vaerenbergh $\mathrm{K}$, Van den Abeele AM, et al. The preanalytical optimization of blood cultures: a review and the clinical importance of benchmarking in 5 Belgian hospitals. Diagn Microbiol Infect Dis. (2012) 73:1-8. doi: 10.1016/j.diagmicrobio.2012.01.009

150. Schmukler M. Density of blood. The Physics Factbook. (2004). Available online at: https://hypertextbook.com/facts/2004/MichaelShmukler.shtml

151. Thavendiranathan P, Bagai A, Ebidia A, Detsky AS, Choudhry NK. Do blood tests cause anemia in hospitalized patients? J Gen Int Med. (2005) 20:520-4. doi: 10.1111/j.1525-1497.2005.0094.x

152. Grietens KP, Ribera JM, Erhart A, Hoibak S, Ravinetto RM, Gryseels C, et al. Perspective piece doctors and vampires in Sub-Saharan Africa: ethical challenges in clinical trial research. Am J Trop Med Hyg. (2014) 91:2135. doi: 10.4269/ajtmh.13-0630

153. Dailey P, Osborn J, Ashley E, Baron E, Dance D, Fusco D, et al. Defining system requirements for simplified blood culture to enable widespread use in resource-limited settings. Diagnostics. (2019) 9:10. doi: 10.3390/diagnostics9010010

154. Frickmann H, Dekker D, Boahen K, Acquah S, Sarpong N, Adu-Sarkodie $\mathrm{Y}$, et al. Increased detection of invasive enteropathogenic bacteria in preincubated blood culture materials by real-time PCR in comparison with automated incubation in Sub-Saharan Africa. Scand J Infect Dis. (2013) 45:616-22. doi: 10.3109/00365548.2013.777777

155. Marks F, Von Kalckreuth V, Aaby P, Adu-sarkodie Y, Ahmed M, Tayeb $\mathrm{E}$, et al. Incidence of invasive salmonella disease in sub-Saharan Africa: a multicentre population-based surveillance study. Lancet Glob Heal. (2017) 5:e310-23. doi: 10.1016/S2214-109X(17)30022-0

156. Weinstein MP, Reller LB, Murphy JR, Lichtenstein KA. The clinical significance of positive blood cultures: a comprehensive analysis of 500 episodes of bacteremia and fungemia in adults. I. Laboratory and epidemiologic observations. Rev Infect Dis. (1983) 5:35-53. doi: 10.1093/clinids/5.1.35

157. Collazos-Blanco A, Perez-Garcia F, Sanchez-Carillo C, de Egea V, Muñoz E, Bouza E. Estimation of missed bloodstream infections without the third blood culture set: a retrospective observational single-centre study. Clin Microbiol Infect. (2019) 25:469-73. doi: 10.1016/j.cmi.2018.06.024

158. Cahill T, Prendergast B. Infective endocarditis. Lancet. (2016) 387:88293. doi: 10.1016/S0140-6736(15)00067-7

159. Li J, Plorde JJ, Carlson LG. Effects of volume and periodicity. Microbiology. (1994) 32:2829-31.

160. Riedel S, Bourbeau P, Swartz B, Brecher S, Carroll KC, Stamper PD, et al. Timing of specimen collection for blood cultures from febrile patients with bacteremia. J Clin Microbiol. (2008) 46:1381-5. doi: 10.1128/JCM. 02033-07

161. Lamy B, Roy P, Flandrois J, Delignette-muller ML. What is the relevance of obtaining multiple blood samples for culture? A comprehensive model to optimize the strategy for diagnosing bacteremia. Clin Infect Dis. (2002) 35:842-50. doi: $10.1086 / 342383$ 
162. Durbin WA, Szymczak EG, Goldmann DA. Quantitative blood cultures in childhood bacteremia. J Pediatr. (1978) 92:7-9. doi: 10.1016/S0022-3476(78)80151-6

163. Kellogg JA, Manzella JP, Bankert DA. Frequency of low-level bacteremia in children from birth to fifteen years of age. J Clin Microbiol. (2000) 38:2181-5.

164. Kellogg J, Ferrentino F, Goodstein M, Liss J, Shapiro S, Banker D. Frequency of low level bacteremia in infants from birth to two months of age. Paediatr Infect Dis J. (1997) 16:381-5. doi: 10.1097/00006454-19970400000009

165. Dietzman D, Fischer G, Schoenknecht F. Neonatal escherichia coli septicemia-bacterial counts in blood. J Pediatr. (1974) 85:128-30. doi: 10.1016/S0022-3476(74)80308-2

166. Isaacman DJ, Karasic RB, Reynolds EA, Kost SI. Effect of number of blood cultures and volume of blood on detection of bacteremia in children. $J$ Pediatr. (1996) 128:190-5. doi: 10.1016/S0022-3476(96)70388-8

167. Kaditis AG, O'Marcaigh AS, Rhodes KH, Weaver AL, Henry NK. Yield of positive blood cultures in pediatric oncology patients by a new method of blood culture collection. Pediatr Infect Dis J. (1996) 15:61520. doi: 10.1097/00006454-199607000-00011

168. Howie SR. Blood sample volumes in child health research: review of safe limits. Bull World Health Organ. (2011) 89:4653. doi: 10.2471/BLT.10.080010

169. Kuijpers LMF, Maltha J, Guiraud I, Kaboré B, Lompo P, Devlieger H, et al. Severe anaemia associated with Plasmodium falciparum infection in children: consequences for additional blood sampling for research. Malar J. (2016) 15:304. doi: 10.1186/s12936-016-1356-9

170. Schelonka RL, Chai MK, Yoder BA, Hensley D, Brockett RM, Ascher DP. Volume of blood required to detect common neonatal pathogens. J Pediatr. (1996) 129:275-8. doi: 10.1016/S0022-3476(96)70254-8

171. Harewood FC, Curtis N, Daley AJ, Bryant PA, Gwee A, Connell TG. Adequate or Inadequate? The volume of blood submitted for blood culture at a tertiary children's hospital. Clin Pediatr (Phila). (2018) 3:18. doi: $10.1177 / 0009922818778042$

172. Bard JD, Tekippe M. Diagnosis of bloodstream infections in children. J Clin Microbiol. (2016) 54:1418-24. doi: 10.1128/JCM.02919-15

173. Hall KK, Lyman JA. Updated review of blood culture contamination. Clin Microbiol Rev. (2006) 19:788-802. doi: 10.1128/CMR.00062-05

174. Weinstein MP. Blood culture contamination: persisting problems and partial progress. J Clin Microbiol. (2003) 41:22758. doi: 10.1128/JCM.41.6.2275-2278.2003

175. García P, Benítez R, Lam M, Salinas AM, Wirth H, Espinoza C, et al. Coagulase-negative staphylococci: clinical, microbiological and molecular features to predict true bacteraemia. J Med Microbiol. (2004) 53:6772. doi: 10.1099/jmm.0.04994-0

176. Pien BC, Sundaram P, Raoof N, Costa SF, Mirrett S, Woods CW, et al. The clinical and prognostic importance of positive blood cultures in adults. Am J Med. (2010) 123:819-28. doi: 10.1016/j.amjmed.2010.03.021

177. Dawson S. Blood culture contaminants. J Hosp Infect. (2014) 87:110. doi: 10.1016/j.jhin.2014.02.009

178. Abrahams MS, Whitelaw AC, Orth H. Time for a culture change? Suboptimal compliance with blood culture standards at a district hospital in Cape Town. South African Med J. (2015) 105:1039-43. doi: 10.7196/SAMJ.2015.v105i12.9442

179. Mtunthama N, Gordon SB, Kusimbwe T, Zijlstra EE, Molyneux ME, French $\mathrm{N}$. Blood culture collection technique and pneumococcal surveillance in Malawi during the four year period 2003-2006: an observational study. BMC Infect Dis. (2008) 8:1-6. doi: 10.1186/1471-2334-8-137

180. Nkrumah NO, Labi AK, Addison NO, Ewuramma J, Labi M, Mensah GA. Trends in paediatric and adult bloodstream infections at a Ghanaian referral hospital: a retrospective study. Ann Clin Microbiol Antimicrob. (2016) 15:110. doi: $10.1186 / \mathrm{s} 12941-016-0163-\mathrm{z}$

181. Hill PC, Onyeama CO, Ikumapayi UNA, Secka O, Ameyaw S, Simmonds N, et al. Bacteraemia in patients admitted to an urban hospital in West Africa. BMC Infect Dis. (2007) 7:1-8. doi: 10.1186/1471-2334-7-2

182. Nourmoradi H, Moradnejadi K, Moghadam FM, Khosravi B. The effect of dust storm on the microbial quality of ambient air in Sanandaj: a city located in the West of Iran. Glob J Health Sci. (2015) 7:1149. doi: $10.5539 /$ gjhs.v7n7p114
183. Itoga M, Inoue F, Saito N, Kayaba H. Increase in Bacillus spp.-positive blood culture specimens linked to inadequate linen management. Kansenshogaku Zasshi. (2016) 90:480-5. doi: 10.11150/kansenshogakuzasshi.90.480

184. Murni IK, Duke T, Daley AJ, Kinney S, Soenarto Y. True pathogen or contamination: validation of blood cultures for the diagnosis of nosocomial infections in a developing country. J Trop Pediatr. (2018) 64:389-94.

185. Alahmadi YM, Aldeyab MA, McElnay JC, Scott MG, Darwish Elhajji FW, Magee FA, et al. Clinical and economic impact of contaminated blood cultures within the hospital setting. J Hosp Infect. (2011) 77:2336. doi: 10.1016/j.jhin.2010.09.033

186. Zwang O, Albert R. Analysis of strategies to improve cost effectiveness of blood cultures. J Hosp Med. (2006) 1:272-6. doi: 10.1002/jhm.115

187. Bates D, Goldman L, Lee T. Contaminant blood cultures and resource utilization. The true consequences of false-positive results. JAMA. (1991) 265:365-9. doi: 10.1001/jama.1991.03460030071031

188. Lee C, Lin W, Shih H, Wu C, Chen P, Lee H, et al. Clinical significance of potential contaminants in blood cultures among patients in a medical center. J Microbiol Immunol Infect. (2007) 40:438-44.

189. WHO. WHO Guidelines on Drawing Blood: Best Practices in Phlebotomy. World Heal Organisation (2010). p. 1-105.

190. Chapman AK, Aucott SW, Milstone AM. Safety of chlorhexidine gluconate used for skin antisepsis in the preterm infant. J Perinatol. (2012) 32:49. doi: 10.1038/jp.2011.148

191. Tamma PD, Aucott SW, Milstone AM. Chlorhexidine use in the neonatal intensive care unit: results from a national survey. Infect Control Hosp Epidemiol. (2010) 31:846-9. doi: 10.1086/655017

192. Chapman AK, Aucott SW, Gilmore MM, Advani S, Clarke W, Milstone AM. Absorption and tolerability of aqueous chlorhexidine gluconate used for skin antisepsis prior to catheter insertion in preterm neonates. J Perinatol. (2014) 33:768-71. doi: 10.1038/jp.2013.61

193. Schifman RB, Strand CL, Meier FA, Howanitz PJ. Blood culture contamination: a College of American Pathologists Q-Probes study involving 640 institutions and 497134 specimens from adult patients. Arch Pathol Lab Med. (1998) 122:216-21.

194. Strand CL, Wajsbort RR, Sturmann K. Effect of iodophor vs iodine tincture skin preparation of blood culture contamination rate. JAMA. (1993) 269:1004-6. doi: 10.1001/jama.1993.03500080052031

195. Caldeira D, David C, Sampaio C. Skin antiseptics in venous puncturesite disinfection for prevention of blood culture contamination: systematic review with meta-analysis. J Hosp Infect. (2011) 77:223-32. doi: 10.1016/j.jhin.2010.10.015

196. Maiwald M, Chan ESY. The forgotten role of alcohol: a systematic review and meta-analysis of the clinical efficacy and perceived role of chlorhexidine in skin antisepsis. PLoS Negl Trop Dis. (2012) 7:e44277. doi: 10.1371/journal.pone.0044277

197. Mermel LA, Allon M, Bouza E, Craven DE, Flynn P, Grady NPO, et al. Clinical practice guidelines for the diagnosis and management of intravascular catheter-related infection: 2009 update by the Infectious Diseases Society of America. Clin Infect Dis. (2009) 49:1-45. doi: 10.1086/599376

198. Qamruddin A, Khanna N, Orr D. Peripheral blood culture contamination in adults and venepuncture technique: prospective cohort study. BMJ Clin Pathol. (2008) 61:509-13. doi: 10.1136/jcp.2007.047647

199. Mimoz O, Karim A, Mercat A, Cosseron M, Falissard B, Parker F, et al Chlorhexidine compared with povidone-iodine as skin preparation before blood culture. A randomized, controlled trial. Ann Int Med. (1999) 131:8347. doi: 10.7326/0003-4819-131-11-199912070-00006

200. Liu W, Duan Y, Cui W, Li L, Wang X, Dai H, et al. Skin antiseptics in venous puncture site disinfection for preventing blood culture contamination: a Bayesian network meta-analysis of randomized controlled trials. Int J Nurs Stud. (2016) 59:156-62. doi: 10.1016/j.ijnurstu.2016.04.004

201. Maiwald M. Skin antiseptics in venous puncture site disinfection for preventing blood culture contamination: a Bayesian network metaanalysis of randomized controlled trials. Int J Nurs Stud. (2017) 75:912. doi: 10.1016/j.ijnurstu.2017.07.011

202. Weber DJ, Rutala WA, Sickbert-Bennett EE. Outbreaks associated with contaminated antiseptics and disinfectants. Antimicrob Agents Chemother. (2007) 51:4217-24. doi: 10.1128/AAC.00138-07 
203. Ramirez-Arcos S, Goldman M. Skin disinfection methods: prospective evaluation and postimplementation results. Transfusion. (2010) 50:5964. doi: 10.1111/j.1537-2995.2009.02434.x

204. Webster J, Sem B, Foxlee R. Skin preparation with alcohol versus alcohol followed by any antiseptic for preventing bacteraemia or contamination of blood for transfusion (review). Cochrane Database Syst Rev. (2015) CD007948. doi: 10.1002/14651858.CD007948.pub3

205. McDonnell GMC, Russell A. Antiseptics and disinfectants: activity, action, and resistance. Clin Microbiol Rev. (1999) 12:147-79. doi: 10.1128/CMR.12.1.147

206. Bekeris LG, Tworek JA, Walsh MK, Valenstein PN. Trends in blood culture contamination a college of American pathologists Q-tracks study of 356 institutions. Arch Pathol Lab Med. (2005) 129:1222-5. doi: 10.1043/15432165(2005)129[1222:TIBCCA]2.0.CO;2

207. Snyder SR, Favoretto AM, Baetz RA, Derzon JH, Madison BM, Mass D, et al. Effectiveness of practises to reduce culture contamination: a laboratory medicine best practices systematic review and meta-analysis. Clin Biochem. (2012) 45:999-1011. doi: 10.1016/j.clinbiochem.2012.06.007

208. Weightman NC, Kerr KG. Blood culture contamination: having your cake and eating it. J Hosp Infect. (2012) 80:101-2. doi: 10.1016/j.jhin.2011.08.023

209. Bamber A, Cunniffe J, Nayar D, Ganguli R, Falconer E. Effectiveness of introducing blood culture collection packs to reduce contamination rates. Br J Biomed Sci. (2009) 66:6-9. doi: 10.1080/09674845.2009.11730236

210. Dhillon R, Clark J, Azadian B. Reducing blood culture contamination. Hosp Infect Soc. (2009) 73:4-6. doi: 10.1016/j.jhin.2009.06.002

211. Patton RG, Schmitt T. Innovation for reducing blood culture contamination: initial specimen diversion technique. J Clin Microbiol. (2010) 48:45013. doi: 10.1128/JCM.00910-10

212. Binkhamis K, Forward K. Effect of the initial specimen diversion technique on blood culture. J Clin Microbiol. (2014) 52:980-1. doi: 10.1128/JCM.02773-13

213. Rupp ME, Cavalieri RJ, Marolf C, Lyden E. Reduction in blood culture contamination through use of initial specimen diversion device. Clin Infect Dis. (2017) 65:201-5. doi: 10.1093/cid/cix304

214. Hoffman PC, Arnow PM, Goldmann DA, Parrott PL, Stamm WE, McGowan JE. False-positive blood cultures association with nonsterile blood collection tubes. JAMA. (1976) 236:2073-5. doi: 10.1001/jama.1976.03270190029023

215. Simor A, Ricci J, Lau A, Bannatyne R, Ford-Jones L. Pseudobacteremia due to pseudomonas fluorescens. Paediatr Infect Dis J. (1985) 4:50812. doi: 10.1097/00006454-198509000-00014

216. Mcneil MM, Davis BJ, Anderson RL, Martone WJ, Solomon SL. Mechanism of cross-contamination of blood culture bottles in outbreaks of pseudobacteremia associated with nonsterile blood collection tubes. $J$ Clincial Microbiol. (1985) 22:23-5.

217. Aronson MD, Bor DH. Diagnostic decision: blood cultures. Ann Int Med. (1987) 106:246-53. doi: 10.7326/0003-4819-106-2-246

218. Dargère S, Parienti JJ, Roupie E, Gancel PE, Wiel E, Smaiti N, et al. Unique blood culture for diagnosis of bloodstream infections in emergency departments: a prospective multicentre study. Clin Microbiol Infect. (2014) 20:O920-7. doi: 10.1111/1469-0691.12656

219. Arendrup M, Jensen IP, Justesen T. Diagnosing bacteremia at a danish hospital using one early large blood volume for culture. Scand J Infect Dis. (1996) 28:609-14. doi: 10.3109/00365549609037969

220. Tonnesen A, Martin P, Lockwood WR. Cultures of blood drawn by catheters vs venipuncture. JAMA. (1976) 235:1877. doi: 10.1001/jama.1976.03260430047025

221. Isaacman D, Karasic R. Utility of collecting blood cultures through newly inserted intravenous catheters. Paediatr Infect Dis J. (1990) 9:8158. doi: 10.1097/00006454-199011000-00007

222. Kelly A, Klim S. Taking blood cultures from a newly established intravenous catheter in the emergency department does not increase the rate of contaminated blood cultures. Emerg Med Australas. (2013) 25:4358. doi: 10.1111/1742-6723.12121

223. Smart D, Baggoley C, Head J, Noble D, Wetherall B, Gordon D. Effect of needle changing and intravenous cannula collection on blood culture contamination rates. Ann Emerg Med. (1993) 22:1164-8. doi: 10.1016/S0196-0644(05)80983-7
224. Norberg A, Christopher N, Ramundo M, Bower J, Berman S. Contamination rates of blood cultures obtained by dedicated phlebotomy vs intravenous catheter. JAMA. (2003) 289:726-9. doi: 10.1001/jama.289.6.726

225. Weddle G, Jackson M, Selvarangan R. Reducing blood culture contamination in a pediatric emergency department. Paediatr Emerg Care. (2011) 27:17981. doi: 10.1097/PEC.0b013e31820d652b

226. Self WH, Speroff T, McNaughton CD, Wright PW, Miller G, Johnson JG, et al. Blood culture collection through peripheral intravenous catheters increases the risk of specimen contamination among adult emergency department patients. Infect Control Hosp Epidemiol. (2012) 33:5246. doi: $10.1086 / 665319$

227. Ramsook C, Childers K, Cron S, Nirken M. Comparison of bloodculture contamination rates in a pediatric emergency room: newly inserted intravenous catheters versus venipuncture. Infect Control Hosp Epidemiol. (2000) 21:649-51. doi: 10.1086/501708

228. Hall RT, Domenico HJ, Self WH, Hain PD. Reducing the blood culture contamination rate in a pediatric emergency department and subsequent cost savings abstract. Paediatrics. (2013) 131:e292-7. doi: 10.1542/peds.2012-1030

229. Spivack ML, Shannon R, Natsios GA, Wood J. Two epidemics of pseudobacteremia due to Staphylococcus aureus and Aerococcus viridans. Infect Control. (1980) 1:321-3. doi: 10.1017/S0195941700 053261

230. Boutros N, Gonullu N, Casetta A, Guibert M, Ingrand D, Lebrun L. Ralstonia pickettii traced in blood culture bottles. J Clin Microbiol. (2002) 40:26667. doi: 10.1128/JCM.40.7.2666-2667.2002

231. Altindis M, Koroglu M, Demiray T, Dal T, Ozdemir M, Sengil AZ, et al. A multicenter evaluation of blood culture practices, contamination rates, and the distribution of causative bacteria. Jundishapur J Microbiol. (2016) 9:9-14. doi: 10.5812/jjm.29766

232. Kim N-H, Kim M, Lee S, Yun NR, Kim K-H, Park SW, et al. Effect of routine sterile gloving on contamination rates in blood culture. Ann Intern Med. (2011) 154:145-52. doi: 10.7326/0003-4819-154-3-201102010-00003

233. Self WH, Mickanin J, Grijalva CG, Grant FH, Henderson MC, Corley G, et al. Reducing blood culture contamination in community hospital emergency departments: multicenter evaluation of a quality improvement intervention wesley. Acad Emerg Med. (2014) 21:274-82. doi: 10.1111/acem.12337

234. Jagger J, Perry J. Risky phlebotomy with a syringe. Nursing (Lond). (2001) 31:73. doi: 10.1097/00152193-200131020-00031

235. Spitalnic SJ, Woolard RH, Mermel LA. The significance of changing needles when inoculating blood cultures: a meta-analysis. Clin Infect Dis. (1995) 21:1103-6. doi: 10.1093/clinids/21.5.1103

236. M'ikhanata NM, Lynfield R, Van Beneden CA, de Valk H. Infectious Disease Surveillance. 2nd ed. Hoboken: Wiley-Blackwell (2013).

237. Hawkins RC. Laboratory Turnaround Time. Q14Clin Biochem Rev. (2007) 28:179-94.

238. Szymczak EG, Barr JT, Durbin WA, Goldmann DA. Evaluation of blood culture procedures in a pediatric hospital. J Clin Microbiol. (1979) 9:88-92.

239. Ganguli LA, O'Hare W, Hyde W. Rapid detection of bacteraemia by early subculture. J Med Microbiol. (1984) 17:311-5. doi: 10.1099/00222615-17-3-311

240. Todd J, Roe M. Rapid detection of bacteremia by an early subculture technic. Am J Clin Pathol. (1975) 64:694-9. doi: 10.1093/ajcp/64. 5.694

241. Harkness JL, Hall M, Ilstrup DM, Washington JA. Effects of atmosphere of incubation and of routine subcultures on detection of bacteremia in vacuum blood culture bottles. J Clin Microbiol. (1975) 2:296-9.

242. Sliva HS, Washington JA. Optimal time for routine early subculture of blood cultures. J Clin Microbiol. (1980) 12:445-6.

243. Bourbeau PP, Heiter BJ, Naumovitz DW. Nonvalue of terminal aerobic subculture of unvented Roche Columbia broth blood culture bottles. J Clin Microbiol. (1992) 30:495-6.

244. Campbell J, Washington JA. Evaluation of the necessity for routine terminal subcultures of previously negative blood cultures. J Clin Microbiol. (1980) 12:576-8

245. Gill VJ. Lack of clinical relevance in routine terminal subculturing of blood cultures. J Clincial Microbiol. (1981) 14:116-8. 
246. Kiehn TE, Wong B, Edwards FF, Armstrong D. Routine aerobic terminal subculturing of blood cultures in a cancer hospital. J Clin Microbiol. (1983) 18:885-9.

247. Blazevic DJ, Stemper JE, Matsen JM. Comparison of macroscopic examination, routine gram stains, and routine subcultures in the initial detection of positive blood cultures. Appl Microbiol. (1974) 27:537-9.

248. Mirrett S, Lauer BA, Miller GA, Reller LB. Comparison of acridine orange, methylene blue, and gram stains for blood cultures. J Clin Microbiol. (1982) 15:562-6.

249. Lambregts MMC, Warreman EB, Bernards AT, Veelken JH, von dem Borne PA, Dekkers OM, et al. Distribution and clinical determinants of time-topositivity of blood cultures in patients with neutropenia. Eur J Haematol. (2017) 100:206-14. doi: 10.1111/ejh.13001

250. Alonso C, Rello J, Mirelis B, Pericas R, Navarro F, Prats G. Comparison of the automatized BacT/ALERT blood culture incubation and reading system and a conventional system. Enferm Infecc Microbiol Clin. (1995) 13:17-22.

251. Lambregts MMC, Bernards AT, Van Der Beek MT, Visser G, De Boer MG. Time to positivity of blood cultures supports early re-evaluation of empiric broad-spectrum antimicrobial therapy. PLoS ONE. (2019) 14:e0208819. doi: 10.1371/journal.pone.0208819

252. Nasir AA, Babalola OM. Clinical spectrum of discharges against medical advice in a developing country figure 1: Age and Gender distribution. Indian J Surg. (2008) 70:68-72. doi: 10.1007/s12262-008-0018-8

253. Onyiriuka A. Discharge of hospitalized under-fives against medical advice in Benin City, Nigeria. Niger J Clin Pr. (2007) 10:200-4.

254. Tuijn CJ, Msoka E, Mushi DL, Sumari-de Boer M, Chilongola J, van den Broek A. The interface between clinicians and laboratory staff: a field study in northern Tanzania. Afr J Lab Med. (2014) 3:1-7. doi: 10.4102/ajlm.v3i1.126

255. Moyo K, Porter C, Chilima B, Mwenda R, Kabue M, Zungu L, et al. Use of laboratory test results in patient management by clinicians in Malawi. Afr J Lab Med. (2015) 4:1-8. doi: 10.4102/ajlm.v4i1.277

256. Kim MJ, Gottschall RL, Schwabe LD, Randall EL. Effect of agitation and frequent subculturing on recovery of aerobic and facultative pathogens by Roche Septi-Chek and BACTEC blood culture systems. J Clin Microbiol. (1987) 25:312-5.

257. Hawkins BL, Peterson EM, de la Maza LM. Improvement of positive blood culture detection by agitation. Diagn Microbiol Infect Dis. (1986) 5:20713. doi: 10.1016/0732-8893(86)90003-9

258. Weinstein MP, Mirrett S, Reimer LG, Reller LB. Effect of altered headspace atmosphere on yield and speed of detection of the oxoid signal blood culture system versus the BACTEC radiometric system. J Clin Microbiol. (1990) 28:795-7.

259. Mirrett S, Everts RJ, Reller LB. Controlled comparison of original vented aerobic FAN medium with new nonvented BacT/Alert FA medium for culturing blood. J Clin Microbiol. (2001) 39:2098-101. doi: 10.1128/JCM.39.6.2098-2101.2001

260. Ilstrup D, Washington JI. Effects of atmosphere of incubation on recovery of bacteria and yeasts from blood cultures in Tryptic soy broth. Diagn Microbiol Infect Dis. (1983) 1:215-9. doi: 10.1016/0732-8893(83)90020-2

261. Youngs ER, Roberts C. Earlier detection of bacteraemia using conventional microbiological techniques. J Clin Pathol. (1985) 38:593-4. doi: 10.1136/jcp.38.5.593

262. Saito T, Ilinuma Y, Takakura S, Nagao M, Matsushima A, Shirano M, et al. Delayed insertion of blood culture bottles into automated continuously monitoring blood culturesystems increases the time from blood sample collection to the detection of microorganisms in bacteremic patients. J Infect Chemother. (2009) 15:49-53. doi: 10.1007/s10156-008-0664-6

263. Venturelli C, Righi E, Borsari L, Aggazzotti G, Busani S, Mussini C, et al. Impact of pre-analytical time on the recovery of pathogens from blood cultures: results from a large retrospective survey. PLoS ONE. (2017) 12:111. doi: 10.1371/journal.pone.0169466

264. Kerremans JJ, Van Der Bij AK, Goessens W, Verbrugh HA, Vos MC. Needle-to-incubator transport time: logistic factors influencing transport time for blood culture specimens. J Clin Microbiol. (2009) 47:81922. doi: 10.1128/JCM.01829-08

265. Baron EJ, Miller JM, Weinstein MP, Richter SS, Gilligan PH, Thomson $\mathrm{RB}$, et al. A guide to utilization of the microbiology laboratory for diagnosis of infectious diseases: 2013 recommendations by the Infectious
Diseases Society of America (IDSA) and the American Society for Microbiology (ASM). Clin Infect Dis. (2013) 57:485-8. doi: 10.1093/cid/c it441

266. Kirn TJ, Weinstein MP. Update on blood cultures: how to obtain, process, report, and interpret. Clin Microbiol Infect. (2013) 19:51320. doi: 10.1111/1469-0691.12180

267. Van Der Velden LB, Vos FJ, Mouton JW, Sturm PD. Clinical impact of preincubation of blood cultures at $37^{\circ}$ C. J Clin Microbiol. (2011) 49:27580. doi: 10.1128/JCM.00552-10

268. Seegmüller I, Eschenbach U, Kamereck K, Miethke T. Sensitivity of the BacT/ALERT FA-medium for detection of Pseudomonas aeruginosa in preincubated blood cultures and its temperature-dependence. J Med Microbiol. (2004) 53:869-74. doi: 10.1099/jmm.0.45533-0

269. Lemming L, Holt HM, Petersen IS, Østergaard C, Bruun B. Bactec 9240 blood culture system: to preincubate at $35^{\circ} \mathrm{C}$ or not? Clin Microbiol Infect. (2004) 10:1089-91. doi: 10.1111/j.1469-0691.2004.00969.x

270. Klaerner HG, Eschenbach U, Kamereck K, Lehn N, Wagner H, Miethke T. Failure of an automated blood culture system to detect nonfermentative gram-negative bacteria. J Clin Microbiol. (2000) 38:1036-41.

271. Ozen N, Ogunc D, Mutlu D, Ongut G, Baysan B, Gunseren F. Comparison of four methods for rapid identification of Staphylococcus aureus directly from BACTEC 9240 blood culture system. Indian J Med Microbiol. (2011) 29:42-6. doi: 10.4103/0255-0857.76523

272. Thirunavukkarasu S, Rathish KC. Evaluation of direct tube coagulase test in diagnosing staphylococcal bacteremia. J Clin Diagnostic Res. (2014) 8:19-21. doi: 10.7860/JCDR/2014/6687.4371

273. Andriesse GI, Elberts S, Vrolijk A, Verhulst C, Kluytmans JAJW. Evaluation of a fourth-generation latex agglutination test for the identification of Staphylococcus aureus. Eur J Clin Microbiol Infect Dis. (2011) 30:25964. doi: 10.1007/s10096-010-1080-2

274. Dhiman N, Trienski TL, Di Persio LP, Di Persio JR. Evaluation of the BinaxNOW Staphylococcus aureus test for rapid identification of grampositive Cocci from VersaTREK blood culture bottles. J Clin Microbiol. (2013) 51:2939-42. doi: 10.1128/JCM.01087-13

275. Peeters M, Chung P, Lin H, Mortelmans K, Phe C, San C, et al. Diagnostic accuracy of the InBiOS AMD rapid diagnostic test for the detection of Burkholderia pseudomallei antigen in grown blood culture broth. Eur J Clin Microbiol Infect Dis. (2018) 37:1169-77. doi: 10.1007/s10096-018-3237-3

276. Kuijpers LMF, Chung P, Peeters M, Phoba MF, Kham C, Barbé B, et al. Diagnostic accuracy of antigen-based immunochromatographic rapid diagnostic tests for the detection of Salmonella in blood culture broth. PLoS ONE. (2018) 13:1-16. doi: 10.1371/journal.pone.0194024

277. Romero-Gomez MP, Quiles-Melero I, Navarro C, Pano-Pardo JR, GomezGil R, Mingorance J. Evaluation of the BinaxNOW PBP2a assay for the direct detection of methicillin resistance in Staphylococcus aureus from positive blood culture bottles. Diagn Microbiol Infect Dis. (2012) 72:2824. doi: 10.1016/j.diagmicrobio.2011.11.012

278. Cuellar-Rodríguez J, Ponce-De-León A, Quiroz-Mejía R, Galindo-Fraga A, Rolón-Montes-de-Oca A, Hernández-Durán M, et al. Rapid detection of ESBL-producing gram-negative bacteria isolated from blood: a reasonable and reliable tool for middle and low resource countries. Rev Invest Clin. (2009) 61:306-12.

279. Dortet L, Bréchard L, Poirel L, Nordmann P. Rapid detection of carbapenemase-producing Enterobacteriaceae from blood cultures. Clin Microbiol Infect. (2014) 20:340-4. doi: 10.1111/1469-0691.12318

280. Mori M, Ravinetto R, Jacobs J. Quality of medical devices and in vitro diagnostics in resource-limited settings. Trop Med Int Heal. (2011) 16:143949. doi: 10.1111/j.1365-3156.2011.02852.x

281. Rugera SP, McNerney R, Poon AK, Akimana G, Mariki RF, Kajumbula $\mathrm{H}$, et al. Regulation of medical diagnostics and medical devices in the East African community partner states. BMC Health Serv Res. (2014) 14:17. doi: 10.1186/s12913-014-0524-2

282. Xu K, Soucat A, Kutzin J, Brindley C. Public Spending on Health: A Closer Look at Global Trends. World Health Organization (2018).

283. Wagstaff A, Flores G, Hsu J, Smitz M, Chepynoga K, Buisman LR, et al. Progress on catastrophic health spending in 133 countries: a retrospective observational study. Lancet Glob Heal. (2018) 6:16979. doi: 10.1016/S2214-109X(17)30429-1 
284. Ayieko P, Akumu AO, Griffiths UK, English M. The economic burden of inpatient paediatric care in Kenya: household and provider costs for treatment of pneumonia, malaria and meningitis. Cost Eff Resour Alloc. (2009) 7:3. doi: 10.1186/1478-7547-7-3

285. Semret M, Ndao M, Jacobs J, Yansouni C. Point-of-care and point-of'can': leveraging reference-laboratory capacity for integrated diagnosis of fever syndromes in the tropics. Clin Microbiol Infect. (2018) 24:836844. doi: 10.1016/j.cmi.2018.03.044

286. Murray PR, Traynor P, Hopson D. Critical assessment of blood culture techniques: Analysis of recovery of obligate and facultative anaerobes, strict aerobic bacteria, and fungi in aerobic and anaerobic blood culture bottles. $J$ Clin Microbiol. (1992) 30:1462-8.

287. Sharp SE, McLaughlin JC, Goodman JM, Moore J, Spanos SM, Keller DW, et al. Clinical assessment of anaerobic isolates from blood cultures. Diagn Microbiol Infect Dis. (1993) 17:19-22. doi: 10.1016/0732-8893(93)90064-E

288. Ortiz E, Sande M. Routine use of anaerobic blood cultures: are they still indicated? Am J Med. (2000) 15:445-7. doi: 10.1016/S0002-9343(99)00410-6

289. Chandler M, Morton E, Byrd RJ, Fields C, Roy M. Reevaluation of anaerobic blood cultures in a Veteran population. South Med J. (2000) 93:9868. doi: 10.1097/00007611-200093100-00008

290. Kellogg J. Selection of a clinically satisfactory blood culture system: the utility of anaerobic media. Clin Microbiol Newsl. (1995) 17:1214. doi: 10.1016/S0196-4399(00)80021-5

291. Vena A, Muñoz P, Alcalá L, Fernandez-Cruz A, Sanchez C, Valerio $\mathrm{M}$, et al. Are incidence and epidemiology of anaerobic bacteremia really changing? Eur J Clin Microbiol Infect Dis. (2015) 34:16219. doi: 10.1007/s10096-015-2397-7

292. Urbán E. Five-year retrospective epidemiological survey of anaerobic bacteraemia in a university hospital and rewiew of the literature. Eur $J$ Microbiol Immunol (Bp). (2012) 2:140-7. doi: 10.1556/EuJMI.2.2012.2.7

293. Nagy E, Boyanova L, Justesen US. How to isolate, identify and determine antimicrobial susceptibility of anaerobic bacteria in routine laboratories. Clin Microbiol Infect. (2018) 24:1139-48. doi: 10.1016/j.cmi.2018.02.008

294. Riley JA, Heiter BJ, Bourbeau PP. Comparison of recovery of blood culture isolates from two BacT/ALERT FAN aerobic blood culture bottles with recovery from one FAN aerobic bottle and one FAN anaerobic bottle. J Clin Microbiol. (2003) 41:213-7. doi: 10.1128/JCM.41.1.213-217.2003

295. Bartlett JG, Dick J. The controversy regarding routine anaerobic blood cultures. Am J Med. (2000) 108:505-6. doi: 10.1016/S0002-9343(00)00321-1

296. Lee CS, Tang RB, Chung RL, Chen SJ. Evaluation of different blood culture media in neonatal sepsis. J Microbiol Immunol Infect. (2000) 33:165-8.

297. Dunne WMJ, Tillman J, Havens PL. Assessing the need for anaerobic medium for the recovery of clinically significant blood culture isolates in children. Pediatr Infect Dis. (1994) 13:203-6. doi: 10.1097/00006454-199403000-00007

298. Meessen NEI, van Pampus ECM, Jacobs JA. False-positive blood cultures in a patient with acute myeloid leukemia. Clin Microbiol Infect. (1999) 5:769-70. doi: 10.1111/j.1469-0691.1999.tb00713.x

299. Karahan Z, Mumcuoglu I, Guriz H, Tamer D, Balaban N, Aysev D. PCR evaluation of false-positive signals from two automated blood-culture systems. J Med Microbiol. (2006) 55:53-7. doi: 10.1099/jmm.0.46196-0

300. Qian Q, Tang YW, Kolbert CP, Torgerson CA, Hughes JG, Vetter EA, et al. Direct identification of bacteria from positive blood cultures by amplification and sequencing of the 16S rRNA gene: evaluation of BACTEC 9240 instrument true-positive and false-positive results. J Clin Microbiol. (2001) 39:3578-82. doi: 10.1128/JCM.39.10.3578-3582.2001

301. Aesif SW, Swierzbinski MJ, Keiser JF. Positive blood culture results after Plasmodium falciparum diagnosis. Lab Med. (2014) 45:e89-91. doi: 10.1309/LM8BLDCRH6WT0VPP
302. de Vries JJC, van Assen S, Mulder AB, Kampinga GA. Positive blood culture with Plasmodium falciparum: case report. Am J Trop Med Hyg. (2007) 76:1098-9. doi: 10.4269/ajtmh.2007. 76.1098

303. Fischer G, Longfield R, Hemming V, Valdes-Dapena A, Smith L. Pneumococcal sepsis with false-negative blood cultures. Am J Clin Pathol. (1982) 78:348-50. doi: 10.1093/ajcp/78.3.348

304. Petti CA, Woods CW, Reller LB. Streptococcus pneumoniae antigen test using positive blood culture bottles as an alternative method to diagnose pneumococcal bacteremia. J Clin Microbiol. (2005) 43:25102. doi: 10.1128/JCM.43.5.2510-2512.2005

305. WHO. Laboratory Biosafety Manual. 3rd ed. Geneva: World Health Organization (2004). p. 1-178. Available online at: http://books.google.com/ books?hl=en\&lr=\&id=qVHfjFINjzwC\&oi $=$ fnd\&pg $=$ PP9\&dq=Laboratory + biosafety + manual\&ots=Qw_FLuZChi\&amp;sig=xgd8x9nOBNLIDE5wsHWAZP6Kw0

306. CDC. Biosafety in Microbiological and Biomedical Laboratories. 5th ed. Washington, DC: Center for Disease Control and Prevention (2009).

307. Hottes A, Rusek B, Sharples F. Biosecurity Challenges of the Global Expansion of High-Containment Biological Laboratories. Washington, DC: National Academy Press (2012). p. 1-216.

308. Whistler T, Kaewpan A, Blacksell SD. A biological safety cabinet certification program: experiences in southeast Asia. Appl Biosaf. (2016) 21:1217. doi: $10.1177 / 1535676016661769$

309. CLSI. Clinical Laboratory Safety. Approved Guideline. 2nd ed., Vol. 24. Wayne, IL: Clinical Laboratory Standards Institute (2008).

310. Christner M, Rohde H, Wolters M, Sobottka I, Wegscheider K, Aepfelbacher M. Rapid identification of bacteria from positive blood culture bottles by use of matrix-assisted laser desorption-ionization time of flight mass spectrometry fingerprinting. J Clin Microbiol. (2010) 48:158491. doi: 10.1128/JCM.01831-09

311. Adelberg E. Biosafety in the laboratory: prudent practices for handling and disposal of infectious materials. In: Adelberg E, editor. Biosafety in the Laboratory: Prudent Practices for Handling and Disposal of Infectious Materials, Vol. 31. Washington, DC: National Academy Press (1989). p. 107-109.

312. Buehler SS, Madison B, Snyder SR, Derzon JH, Cornish NE, Saubolle $\mathrm{MA}$, et al. Effectiveness of practices to increase timeliness of providing targeted therapy for inpatients with bloodstream infections: a laboratory medicine best practices systematic review and metaanalysis. Clin Microbiol Rev. (2015) 29:59-103. doi: 10.1128/CMR. 00053-14

313. Bouza E, Sousa D, Munoz P, Rodriguez-Creixems M, Fron C, Lechuz JG. Bloodstream infections: a trial of the impact of different methods of reporting positive blood culture results. Clin Infect Dis. (2004) 39:11619. doi: $10.1086 / 424520$

Conflict of Interest Statement: The authors declare that the research was conducted in the absence of any commercial or financial relationships that could be construed as a potential conflict of interest.

Copyright (c) 2019 Ombelet, Barbé, Affolabi, Ronat, Lompo, Lunguya, Jacobs and Hardy. This is an open-access article distributed under the terms of the Creative Commons Attribution License (CC BY). The use, distribution or reproduction in other forums is permitted, provided the original author(s) and the copyright owner(s) are credited and that the original publication in this journal is cited, in accordance with accepted academic practice. No use, distribution or reproduction is permitted which does not comply with these terms. 